\title{
Well-Posedness and Asymptotic Stability of Solutions to the Bresse System under Cattaneo's Law with Infinite Memories and Time-Varying Delays
}

\author{
Gang $\mathrm{Li}^{*}$ and Xiangyu Kong \\ College of Mathematics and Statistics, Nanjing University of Information Science and Technology, \\ Nanjing 210044, China; 15651559885@163.com \\ * Correspondence: ligang@nuist.edu.cn
}

In this paper, we study a one-dimensional Bresse-Cattaneo system with infinite memories and time-dependent delay term (the coefficient of which is not necessarily positive) in the internal feedbacks. First, it is proved that the system is well-posed by means of the Hille-Yosida theorem under suitable assumptions on the relaxation functions. Then, without any restriction on the speeds of wave propagations, we establish the exponential or general decay result by introducing suitable energy and Lyapunov functionals.

Keywords: well-posedness; asymptotic stability; infinite memory; Cattaneo's law; time-varying delay

AMS Subject Classification (2010): 35B40, 93D15, 93D20.

\section{Introduction}

The Bresse system is known as the circular arch problem (see [16] for details) and is given by the following equations:

$$
\left\{\begin{array}{l}
\rho_{1} \varphi_{t t}=Q_{x}+I N+F_{1}, \\
\rho_{2} \psi_{t t}=M_{x}-Q+F_{2}, \\
\rho_{1} w_{t t}=N_{x}-I Q+F_{3}
\end{array}\right.
$$

where

$$
N=k_{3}\left(w_{x}-I \varphi\right), Q=k_{1}\left(\varphi_{x}+I w+\psi\right), M=k_{2} \psi_{x}
$$

denote the axial force, the shear force and the bending moment, respectively, $\rho_{1}=\rho A, \rho_{2}=$ $\rho I, k_{3}=E A, k_{1}=k^{\prime} G A, k_{2}=E I, I=R^{-1}$. The functions $w, \varphi$ and $\psi$ are the longitudinal, vertical and shear angle displacements, respectively. We use $\rho$ for the density, $E$ for the elastic modulus, $G$ for the shear modulus, $k^{\prime}$ for the shear factor, $A$ for the cross-sectional area, $I$ for the second moment of area of the cross-section, $R$ for the radius of curvature of the beam, $F_{i}(i=1,2,3)$ for the external forces. The arch with elastic structure is widely used in the fields of engineering, architecture, ocean engineering, aviation and others. In particular, the free vibration of elastic structure is a function of its natural property, and it is an important research subject in engineering and Mathematics. In the field of mathematical analysis is interesting to know properties which relate the behavior of the energy associated with the respective dynamic model. For feedback laws, for example, We can ask what conditions of the kinetic model can be obtained from the decay of the energy of the solution. In this sense, the property of stabilization has been studied for dynamic problems in elastic structures translated in terms of partial differential equations. 
In this paper, we investigate a Bresse-Cattaneo system with infinite memories and time-varying delays in the internal feedbacks

$$
\begin{cases}\rho_{1} \varphi_{t t}-k_{1}\left(\varphi_{x}+\psi+I w\right)_{x}-I k_{3}\left(w_{x}-I \varphi\right)+\sigma \theta_{x}+\mu_{1} \varphi_{t} & \\ +\mu_{2} \varphi_{t}\left(x, t-\tau_{1}(t)\right)+\int_{0}^{+\infty} g_{1}(s) \varphi_{x x}(x, t-s) \mathrm{d} s=0, & (x, t) \in(0, L) \times(0,+\infty), \\ \rho_{2} \psi_{t t}-k_{2} \psi_{x x}+k_{1}\left(\varphi_{x}+\psi+I w\right)+\tilde{\mu}_{1} \psi_{t} & \\ +\tilde{\mu}_{2} \psi_{t}\left(x, t-\tau_{2}(t)\right)+\int_{0}^{+\infty} g_{2}(s) \psi_{x x}(x, t-s) \mathrm{d} s=0, & (x, t) \in(0, L) \times(0,+\infty), \\ \rho_{1} w_{t t}-k_{3}\left(w_{x}-I \varphi\right)_{x}+I k_{1}\left(\varphi_{x}+\psi+I w\right)+\tilde{\tilde{\mu}}_{1} w_{t} & \\ +\tilde{\tilde{\mu}}_{2} w_{t}\left(x, t-\tau_{3}(t)\right)+\int_{0}^{+\infty} g_{3}(s) w_{x x}(x, t-s) \mathrm{d} s=0, & (x, t) \in(0, L) \times(0,+\infty), \\ \rho_{3} \theta_{t}+q_{x}+\sigma \varphi_{x t}=0, & (x, t) \in(0, L) \times(0,+\infty), \\ \tau q_{t}+q+\gamma \theta_{x}=0, & (x, t) \in(0, L) \times(0,+\infty),\end{cases}
$$

where $g_{i}: \mathbb{R}_{+} \rightarrow \mathbb{R}_{+}(i=1,2,3)$ are given functions, $\tau_{i}(t)(i=1,2,3)$ are time-varying delays, $\mu_{1}, \tilde{\mu}_{1}, \tilde{\tilde{\mu}}_{1}, I, \sigma, \tau, \gamma, \rho_{i}, k_{i}(i=1,2,3)$ are positive constants, and the infinite integrals in (1.1) represent the infinite memories. This system is subject to the Dirichlet boundary conditions

$$
\varphi(0, t)=\varphi(L, t)=\psi(0, t)=\psi(L, t)=w(0, t)=w(L, t)=\theta(0, t)=\theta(L, t)=0, t \in[0,+\infty)
$$

and to the initial conditions

$$
\begin{cases}\varphi(x,-t)=\varphi_{0}(x, t), \psi(x,-t)=\psi_{0}(x, t), w(x,-t)=w_{0}(x, t), & (x, t) \in[0, L] \times[0,+\infty), \\ \varphi_{t}(x, 0)=\varphi_{1}(x), \psi_{t}(x, 0)=\psi_{1}(x), w_{t}(x, 0)=w_{1}(x), & x \in[0, L], \\ \theta(x, 0)=\theta_{0}(x), q(x, 0)=q_{0}(x), & x \in[0, L], \\ \varphi_{t}\left(x, t-\tau_{1}(0)\right)=f_{0}\left(x, t-\tau_{1}(0)\right), & (x, t) \in[0, L] \times\left[0, \tau_{1}(0)\right], \\ \psi_{t}\left(x, t-\tau_{2}(0)\right)=\tilde{f}_{0}\left(x, t-\tau_{2}(0)\right), & (x, t) \in[0, L] \times\left[0, \tau_{2}(0)\right], \\ w_{t}\left(x, t-\tau_{3}(0)\right)=\tilde{\tilde{f}}_{0}\left(x, t-\tau_{3}(0)\right), & (x, t) \in[0, L] \times\left[0, \tau_{3}(0)\right] .\end{cases}
$$

Before stating our main result, let us first mention some other papers related to the problem we address. During the last few decades, there are many works treating about existence and stabilization of Bresse system. Alabau Boussouira et al. [1] considered a Bresse system with one frictional damping working only on the angle displacement. The authors proved that the exponential decay exists when the velocities of the wave propagations are the same. If the wave speeds are different, they showed that the energy of the system decays polynomially to zero with rates that can be improved by taking more regular initial data. Liu and Rao [18] studied the Bresse system with two different dissipative mechanism, given by two temperatures coupled to the system. The authors established exponential decay rate when the vertical and longitudinal waves have the same speeds of wave propagations. Otherwise, the solution decays polynomially to zero with rates $t^{-4+\epsilon}$ or $t^{-6+\epsilon}$ provided the boundary conditions is DirichletC NeumannCNeumann or DirichletCDirichletCDirichlet type respectively. This results was improved by Fatori and 
Rivera [9], the authors considered the Bresse system with thermal dissipation effective only in one equation of the system and obtained exponential decay result when all the wave speeds are equal. In general, they showed the system is not exponentially stable but that there exists polynomial stability with rates that depend on the wave propagations and the regularity of the initial data. Moreover, they introduced a necessary condition to dissipative the semigroup for the polynomial decay. With respect to asymptotic behavior of solutions for Bresse system with infinte memory, Guesmia and Kafini [14] studied a one-dimensional linear Bresse system with infinite memories acting in the three equations of the system of the form

$$
\left\{\begin{aligned}
\rho_{1} \varphi_{t t}-k_{1}\left(\varphi_{x}+\psi+I w\right)_{x}-I k_{3}\left(w_{x}-I \varphi\right) & \\
& +\int_{0}^{+\infty} g_{1}(s) \varphi_{x x}(x, t-s) \mathrm{d} s=0,(x, t) \in(0, L) \times(0,+\infty) \\
\rho_{2} \psi_{t t}-k_{2} \psi_{x x} & +k_{1}\left(\varphi_{x}+\psi+I w\right) \\
& +\int_{0}^{+\infty} g_{2}(s) \psi_{x x}(x, t-s) \mathrm{d} s=0,(x, t) \in(0, L) \times(0,+\infty) \\
\rho_{1} w_{t t}-k_{3}\left(w_{x}-I \varphi\right)_{x}+I k_{1}\left(\varphi_{x}+\psi+I w\right) & \\
& +\int_{0}^{+\infty} g_{3}(s) w_{x x}(x, t-s) \mathrm{d} s=0,(x, t) \in(0, L) \times(0,+\infty) .
\end{aligned}\right.
$$

The authors established the well-posedness and asymptotic stability results for the system under some conditions imposed into the relaxation functions regardless to the speeds of wave propagations. For more papers concerning infinite memory, we refer to [3, 12, 13, 27].

For stabilization of Timoshenko systems via heat effect, there are some results in recent time. Almeida Júnior [2] considered 1-D thermoelastic Timoshenko beam of the form

$$
\begin{cases}\rho_{1} \varphi_{t t}-k\left(\varphi_{x}+\psi\right)_{x}+\sigma \theta_{x}=0, & (x, t) \in(0, L) \times(0,+\infty), \\ \rho_{2} \psi_{t t}-b \psi_{x x}+k\left(\varphi_{x}+\psi\right)-\sigma \theta=0, & (x, t) \in(0, L) \times(0,+\infty), \\ \rho_{3} \theta_{t}-\gamma \theta_{x x}+\sigma\left(\varphi_{x}+\psi\right)_{t}=0, & (x, t) \in(0, L) \times(0,+\infty)\end{cases}
$$

with two types of boundary (Dirichlet-Dirichlet-Dirichlet or Dirichlet-Neumann-Neumann) conditions and established both exponential and polynomial stability results depending on the wave speeds and the initial data. In the above system, the heat equation is governed by Fourier's law of heat conduction. It is well known that the model using the classic Fourier's law leads to the physical paradox of infinite speed of heat propagation. That is, any local thermal disturbance can have an instantaneous effect everywhere in the medium. However, experiments showed that heat conduction in some dielectric crystals at low temperatures propagates with a finite speed. This phenomenon in dielectric crystals is called second sound. To overcome this drawback, a number of modifications of the basic assumption on the relation between the heat flux and the temperature have been made. One of which is the second sound effects observed experimentally in materials at a very low temperature. This theory suggests replacing the classic Fourier's law

$$
q+\gamma \theta_{x}=0
$$

where $q$ is the heat flux and $\gamma$ is the coefficient of thermal conductivity by a modified law of heat conduction called Cattaneo's law

$$
\tau q_{t}+q+\gamma \theta_{x}=0
$$


where $\tau>0$ represents the relaxation time describing the time lag in the response of the heat flux to a gradient in the temperature. On the basis of the above theory, Santos et al. [28] studied the Timoshenko beam model with second sound of the form

$$
\begin{cases}\rho_{1} \varphi_{t t}-k\left(\varphi_{x}+\psi\right)_{x}=0, & (x, t) \in(0, L) \times(0,+\infty), \\ \rho_{2} \psi_{t t}-b \psi_{x x}+k\left(\varphi_{x}+\psi\right)+\delta \theta_{x}=0, & (x, t) \in(0, L) \times(0,+\infty), \\ \rho_{3} \theta_{t}+q_{x}+\delta \psi_{t x}=0, & (x, t) \in(0, L) \times(0,+\infty), \\ \tau q_{t}+\beta q+\theta_{x}=0, & (x, t) \in(0, L) \times(0,+\infty) .\end{cases}
$$

The authors obtained exponential decay result when the stability number $\chi_{\tau}=0$. Otherwise, the polynomial decay result is obtained. Moreover, they showed that the rate is optimal. For more papers related to the second sound, we refer the reader to $[4,6,25]$ and the references therein.

In recent years, the PDEs with time delays have become an active area of research and arise in many practical problems. The presence of delay may act as a source of instability. In [8], the authors showed that a small delay in a boundary control can destabilize a system which is uniformly asymptotically stable in the absence delays. To stabilize a hyperbolic system involving input delay terms, additional control will be necessary [22, 29]. Kirane and Said-Houari [20] considered a viscoelastic wave equation with a linear damping and a delay of the form

$u_{t t}(x, t)-\Delta u(x, t)+\int_{0}^{t} g(t-s) \Delta u(x, t-s) \mathrm{d} s+\mu_{1} u_{t}(x, t)+\mu_{2} u_{t}(x, t-\tau)=0,(x, t) \in \Omega \times(0, \infty)$,

where $\mu_{1}$ and $\mu_{2}$ are positive constants. They established a general decay result under the condition that $\mu_{2} \leq \mu_{1}$. Later, Liu [15] improved this result by considering the equation with a time-varying delay term, with not necessarily positive coefficient $\mu_{2}$ of the delay term. Moreover, some researchers considered the Timoshenko and Bresse systems with delay term. For instance, Said-Houari and Laskri [26] considered the following Timoshenko system with a constant time delay of the form

$$
\left\{\begin{array}{lr}
\rho_{1} \varphi_{t t}-K\left(\varphi_{x}+\psi\right)_{x}=0, & (x, t) \in(0,1) \times(0,+\infty), \\
\rho_{2} \psi_{t t}-b \psi_{x x}+K\left(\varphi_{x}+\psi\right)+\mu_{1} \psi_{t}(x, t)+\mu_{2} \psi_{t}(x, t-\tau)=0, & (x, t) \in(0,1) \times(0,+\infty) .
\end{array}\right.
$$

They established an exponential decay result for the case of equal-speed wave propagation under the assumption $\mu_{2}<\mu_{1}$. More recently, Kirane el al. [19] considered the following Timoshenko system with a time-varying delay of the form

$$
\left\{\begin{array}{lr}
\rho_{1} \varphi_{t t}-K\left(\varphi_{x}+\psi\right)_{x}=0, & (x, t) \in(0,1) \times(0,+\infty), \\
\rho_{2} \psi_{t t}-b \psi_{x x}+K\left(\varphi_{x}+\psi\right)+\mu_{1} \psi_{t}(x, t)+\mu_{2} \psi_{t}(x, t-\tau(t))=0, & (x, t) \in(0,1) \times(0,+\infty),
\end{array}\right.
$$

where $\tau(t)$ represents the time-varying delay, $0<\tau_{0} \leq \tau(t) \leq \bar{\tau}$ and $\mu_{1}, \mu_{2}$ are positive constants. Under the assumpyions $\mu_{2}<\sqrt{1-d_{1}} \mu_{1}$ and $\tau^{\prime}(t) \leq 1$, they proved the exponential decay result.

Motivated by the above results, we investigate in this paper system (1.1) under suitable assumptions and prove the well-posedness and the asymptotic stability of system.

Using $(1.1)_{5}$ and the boundary condition, we can easily verify that

$$
\frac{d}{d t} \int_{0}^{L} q(x, t) \mathrm{d} x+\frac{1}{\tau} \int_{0}^{L} q(x, t) \mathrm{d} x=0 .
$$


Consequently, we obtain

$$
\int_{0}^{L} q(x, t) \mathrm{d} x=e^{-\frac{t}{\tau}} \int_{0}^{L} q_{0}(x) \mathrm{d} x .
$$

If we set

$$
\bar{q}(x, t) \mathrm{d} x=q(x, t)-\frac{e^{-\frac{t}{\tau}}}{L} \int_{0}^{L} q_{0}(x) \mathrm{d} x,
$$

then simple substitution shows that $(\varphi, \psi, w, \theta, \bar{q})$ satisfies problem (1.1), and we have

$$
\int_{0}^{L} \bar{q}(x, t) \mathrm{d} x=0
$$

From now on, we use the new variables $(\varphi, \psi, w, \theta, \bar{q})$, but we denote them by $(\varphi, \psi, w, \theta, q)$ for simplicity.

The main difficulty in carrying out this paper is the simultaneous appearance of the infinite memories, heat effect and time-varying delay. To overcome this difficulty, we have two key points in the proofs. On the one hand, to create the negative counterparts of the terms in the energy, we combine the fireworks of [4], [14] and [19] with necessary modifications. On the other hand, to estimate the infinite integral terms in (4.20) below, we use the approach which was first proved by Guesmia [10] and used by many researchers (see [11, 14]).

This paper is organized as follows. In section 2, we present some assumptions needed for our work and state the main results. In section 3, we prove the well-posedness of problem (1.1). In section 4 , we prove the asymptotic stability of problem (1.1).

\section{Preliminaries and main results}

In this section, we shall introduce some notation, basic definitions and main results which will be needed in the course of this paper.

First, we assume the following hypotheses:

$$
\begin{gathered}
\tau_{i}(t) \in W^{2, \infty}([0, T]), \quad \forall T>0, i=1,2,3, \\
0<\tau_{01}<\tau_{1}(t)<\tilde{\tau}_{1}, \quad \forall t>0 \\
0<\tau_{02}<\tau_{2}(t)<\tilde{\tau}_{2}, \quad \forall t>0 \\
0<\tau_{03}<\tau_{3}(t)<\tilde{\tau}_{3}, \quad \forall t>0 \\
\tau_{1}^{\prime}(t) \leq d_{1}<1, \quad \forall t>0 \\
\tau_{2}^{\prime}(t) \leq d_{2}<1, \quad \forall t>0 \\
\tau_{3}^{\prime}(t) \leq d_{3}<1, \quad \forall t>0 \\
\left|\mu_{2}\right| \leq \sqrt{1-d_{1}} \mu_{1} \\
\left|\tilde{\mu}_{2}\right| \leq \sqrt{1-d_{2}} \tilde{\mu}_{1} \\
\left|\tilde{\tilde{\mu}}_{2}\right| \leq \sqrt{1-d_{3}} \tilde{\tilde{\mu}}_{1}
\end{gathered}
$$


where $\tau_{01}, \tau_{02}, \tau_{03}, \tilde{\tau}_{1}, \tilde{\tau}_{2}, \tilde{\tau}_{3}, d_{1}, d_{2}, d_{3}$ are positive constants.

Next, let us consider the following variables (see [7]):

$$
\begin{gathered}
\left\{\begin{array}{l}
\eta_{1}(x, t, s)=\varphi(x, t)-\varphi(x, t-s),(x, t, s) \in(0, L) \times(0,+\infty) \times(0,+\infty), \\
\eta_{2}(x, t, s)=\psi(x, t)-\psi(x, t-s),(x, t, s) \in(0, L) \times(0,+\infty) \times(0,+\infty), \\
\eta_{3}(x, t, s)=w(x, t)-w(x, t-s),(x, t, s) \in(0, L) \times(0,+\infty) \times(0,+\infty),
\end{array}\right. \\
\left\{\begin{array}{l}
z_{1}(x, \rho, t)=\varphi_{t}\left(x, t-\tau_{1}(t) \rho\right),(x, \rho, t) \in(0, L) \times(0,1) \times(0,+\infty), \\
z_{2}(x, \rho, t)=\psi_{t}\left(x, t-\tau_{2}(t) \rho\right),(x, \rho, t) \in(0, L) \times(0,1) \times(0,+\infty), \\
z_{3}(x, \rho, t)=w_{t}\left(x, t-\tau_{3}(t) \rho\right),(x, \rho, t) \in(0, L) \times(0,1) \times(0,+\infty) .
\end{array}\right.
\end{gathered}
$$

Then, it is easy to check that

$$
\begin{aligned}
& \begin{cases}\partial_{t} \eta_{1}+\partial_{s} \eta_{1}-\varphi_{t}=0, & (x, t, s) \in(0, L) \times(0,+\infty) \times(0,+\infty), \\
\partial_{t} \eta_{2}+\partial_{s} \eta_{2}-\psi_{t}=0, & (x, t, s) \in(0, L) \times(0,+\infty) \times(0,+\infty), \\
\partial_{t} \eta_{3}+\partial_{s} \eta_{3}-w_{t}=0, & (x, t, s) \in(0, L) \times(0,+\infty) \times(0,+\infty), \\
\eta_{i}(0, t, s)=\eta_{i}(L, t, s)=0, & (t, s) \in[0,+\infty) \times[0,+\infty), i=1,2,3, \\
\eta_{i}(x, t, 0)=0, & (x, t) \in(0, L) \times(0,+\infty), i=1,2,3,\end{cases} \\
& \tau_{i}(t) z_{i t}(x, \rho, t)+\left(1-\tau_{i}^{\prime}(t) \rho\right) z_{i \rho}(x, \rho, t)=0,(x, \rho, t) \in(0, L) \times(0,1) \times(0,+\infty), i=1,2,3 .
\end{aligned}
$$

Therefore, problem (1.1) takes the form:

$$
\begin{cases}\rho_{1} \varphi_{t t}-k_{1}\left(\varphi_{x}+\psi+I w\right)_{x}-I k_{3}\left(w_{x}-I \varphi\right)+\mu_{1} \varphi_{t}+\mu_{2} z_{1}(x, 1, t) & \\ +\int_{0}^{+\infty} g_{1}(s) \varphi_{x x} \mathrm{~d} s-\int_{0}^{+\infty} g_{1}(s) \partial_{x x} \eta_{1}(x, t) \mathrm{d} s+\sigma \theta_{x}=0, & (x, t) \in(0, L) \times(0,+\infty) \\ \rho_{2} \psi_{t t}-k_{2} \psi_{x x}+k_{1}\left(\varphi_{x}+\psi+I w\right)+\tilde{\mu}_{1} \psi_{t}+\tilde{\mu}_{2} z_{2}(x, 1, t) & \\ +\int_{0}^{+\infty} g_{2}(s) \psi_{x x} \mathrm{~d} s-\int_{0}^{+\infty} g_{2}(s) \partial_{x x} \eta_{2}(x, t) \mathrm{d} s=0, & (x, t) \in(0, L) \times(0,+\infty) \\ \rho_{1} w_{t t}-k_{3}\left(w_{x}-I \varphi\right)_{x}+I k_{1}\left(\varphi_{x}+\psi+I w\right)+\tilde{\tilde{\mu}}_{1} w_{t}+\tilde{\tilde{\mu}}_{2} z_{3}(x, 1, t) & \\ +\int_{0}^{+\infty} g_{3}(s) w_{x x} \mathrm{~d} s-\int_{0}^{+\infty} g_{3}(s) \partial_{x x} \eta_{3}(x, t) \mathrm{d} s=0, & (x, t) \in(0, L) \times(0,+\infty) \\ \rho_{3} \theta_{t}+q_{x}+\sigma \varphi_{x t}=0, & (x, t) \in(0, L) \times(0,+\infty) \\ \tau q_{t}+q+\gamma \theta_{x}=0, & (x, \rho, t) \in(0, L) \times(0,1) \times(0,+\infty) \\ \tau_{1}(t) z_{1 t}(x, \rho, t)+\left(1-\tau_{1}^{\prime}(t) \rho\right) z_{1 \rho}(x, \rho, t)=0, & (x, \rho, t) \in(0, L) \times(0,1) \times(0,+\infty) \\ \tau_{2}(t) z_{2 t}(x, \rho, t)+\left(1-\tau_{2}^{\prime}(t) \rho\right) z_{2 \rho}(x, \rho, t)=0, & (x, \rho, t) \in(0, L) \times(0,1) \times(0,+\infty) \\ \tau_{3}(t) z_{3 t}(x, \rho, t)+\left(1-\tau_{3}^{\prime}(t) \rho\right) z_{3 \rho}(x, \rho, t)=0, & \end{cases}
$$


The above system is subject to the following initial and boundary conditions

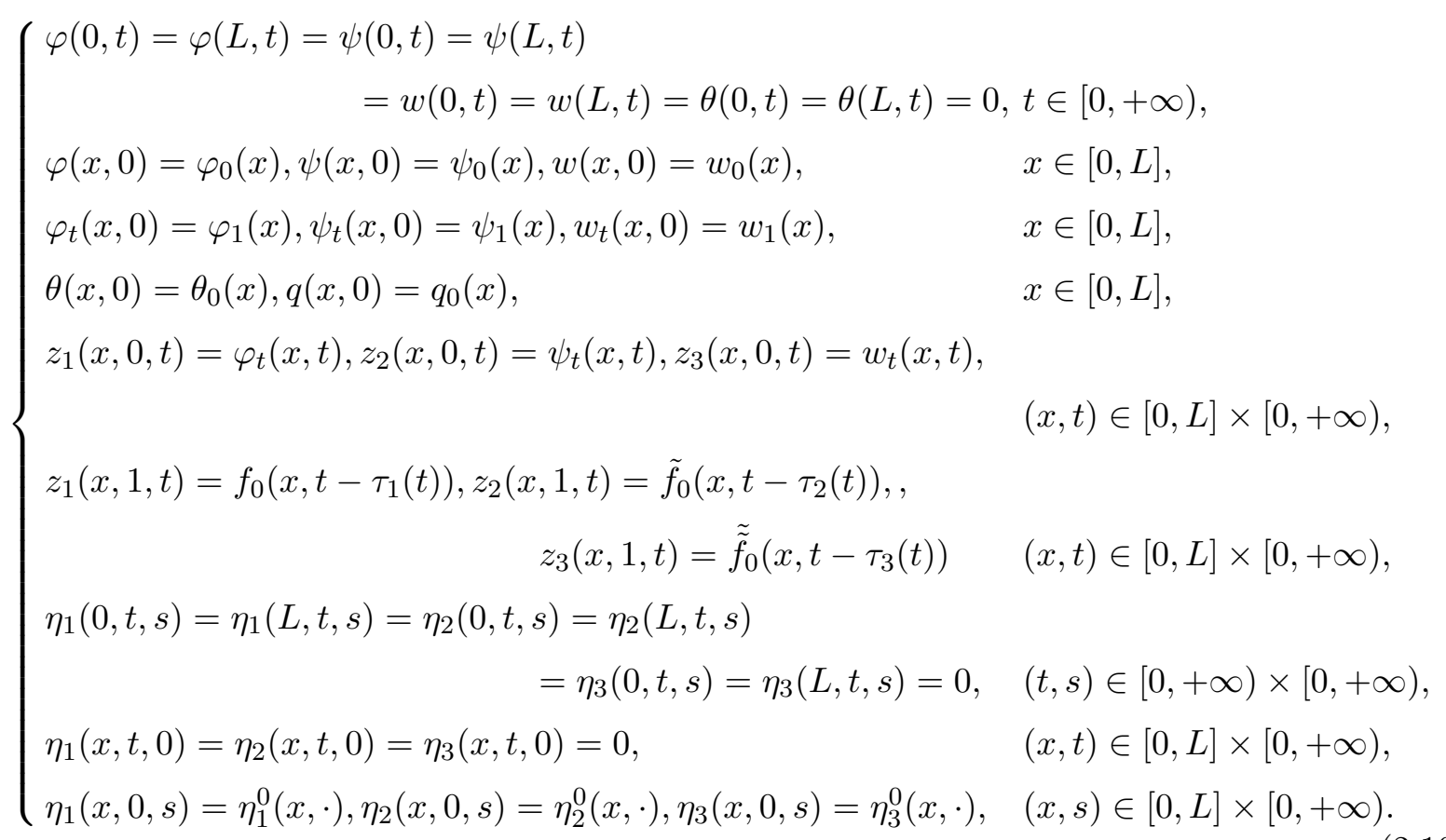

Let

$$
U=\left(\varphi, \psi, w, \theta, q, \varphi_{t}, \psi_{t}, w_{t}, z_{1}, z_{2}, z_{3}, \eta_{1}, \eta_{2}, \eta_{3}\right)^{T}
$$

and

$$
\begin{aligned}
U^{0}(x)= & \left(\varphi_{0}(x, 0), \psi_{0}(x, 0), w_{0}(x, 0), \theta(x, 0), q(x, 0), \varphi_{1}(x), \psi_{1}(x), w_{1}(x),\right. \\
& \left.f\left(\cdot,-\tau_{1}(0) \rho\right), f\left(\cdot,-\tau_{2}(0) \rho\right), f\left(\cdot,-\tau_{3}(0) \rho\right), \eta_{1}^{0}(x, \cdot), \eta_{2}^{0}(x, \cdot), \eta_{3}^{0}(x, \cdot)\right)^{T} .
\end{aligned}
$$

Then problem (2.9)-(2.10) can be written as

$$
\left\{\begin{array}{l}
\partial_{t} U=\mathscr{A} U, \\
U(x, 0)=U^{0}(x),
\end{array}\right.
$$


where time varying operator $\mathscr{A}$ is defined by

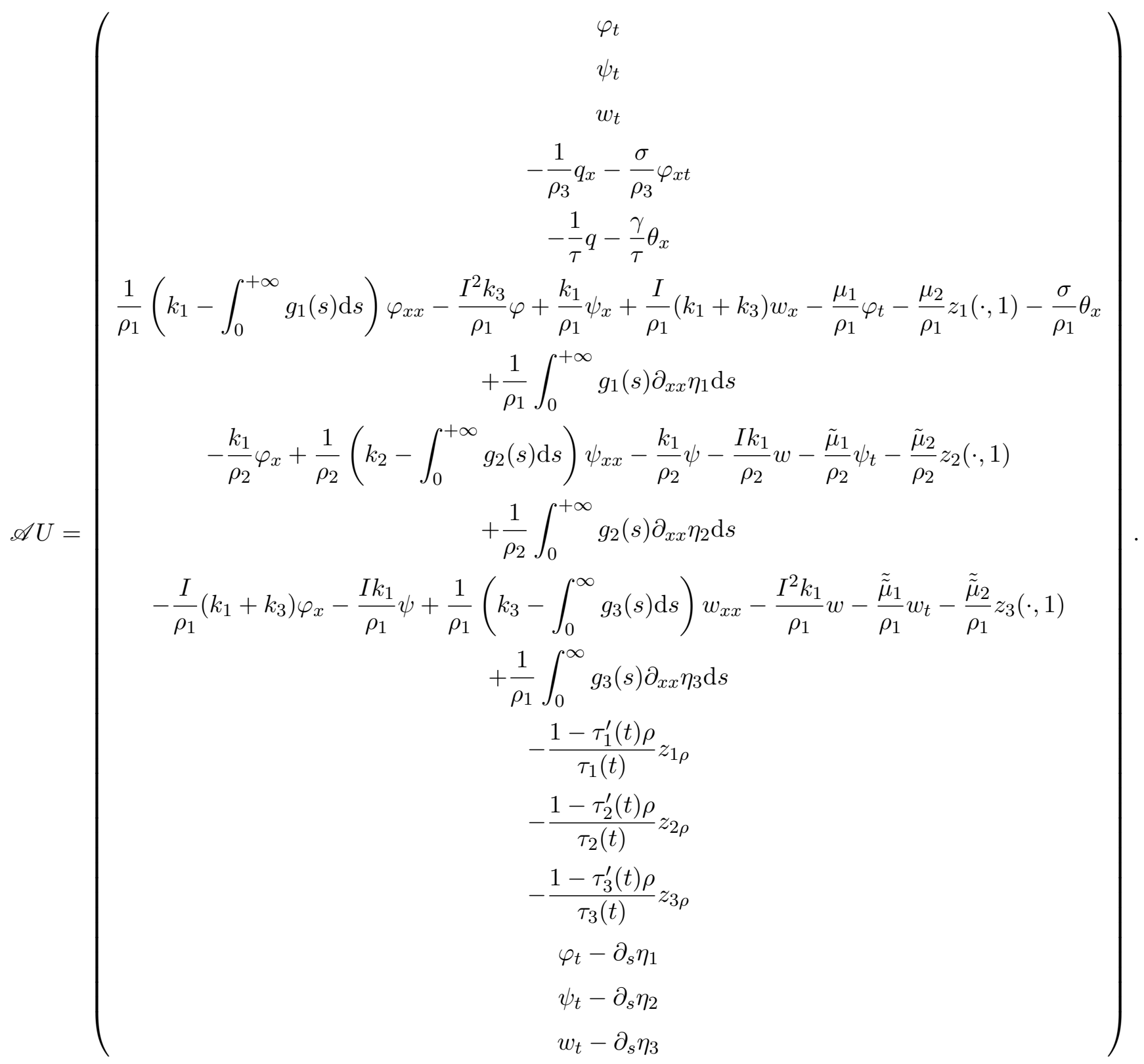

Now, we consider the following space

$$
L_{*}^{2}(0, L)=\left\{\omega \in L^{2}(0, L): \int_{0}^{L} \omega(s) \mathrm{d} s=0\right\}, \quad H_{*}^{1}=H^{1}(0, L) \cap L_{*}^{2}(0, L)
$$

and define the functional spaces of $U$ as follow:

$$
\mathscr{H}=\left(H_{0}^{1}(0, L)\right)^{3} \times L^{2}(0, L) \times L_{*}^{2}(0, L) \times\left(L^{2}(0, L)\right)^{3} \times\left(L^{2}((0,1) \times(0, L))\right)^{3} \times H_{1}^{*} \times H_{2}^{*} \times H_{3}^{*},
$$

where

$$
H_{i}^{*}=\left\{\omega: \mathbb{R}^{+} \longrightarrow H_{0}^{1}(0, L), \int_{0}^{L} \int_{0}^{+\infty} g_{i}(s) \omega_{x}^{2} \mathrm{~d} s \mathrm{~d} x<+\infty\right\}
$$


Then the domain of $\mathscr{A}$ is defined by

$$
\begin{aligned}
D(\mathscr{A})=\{U & \left(H^{2}(0, L) \cap H_{0}^{1}(0, L)\right)^{3} \times H_{0}^{1}(0, L) \times H_{*}^{1}(0, L) \times\left(H_{0}^{1}(0, L)\right)^{3} \\
& \left.\times L^{2}\left((0, L), H^{1}(0, L)\right)^{3} \times \mathscr{H}_{1}^{*} \times \mathscr{H}_{2}^{*} \times \mathscr{H}_{3}^{*}\right\}
\end{aligned}
$$

where

$$
\mathscr{H}_{i}^{*}=\left\{\omega_{i} \in H_{i}^{*}, \partial_{s} \omega_{i} \in H_{i}^{*}, \omega_{i}(x, t, 0)=0\right\} .
$$

For the relaxation functions $g_{i}$, motivated by [14], we have the following assumptions:

(G1) $g_{i}: \mathbb{R}^{+} \rightarrow \mathbb{R}^{+}(i=1,2,3)$ are non-increasing differentiable functions and integrate on $(0,+\infty)$ such that there exists a positive constant $k_{0}$ satisfying, for any $(\varphi, \psi, w) \in H_{0}^{1}(0, L) \times$ $H_{0}^{1}(0, L) \times H_{0}^{1}(0, L)$

$$
\begin{aligned}
k_{0} \int_{0}^{L}\left(\varphi_{x}^{2}+\psi_{x}^{2}+w_{x}^{2}\right) \mathrm{d} x \leq & \int_{0}^{L}\left(k_{2} \psi_{x}^{2}+k_{1}\left(\varphi_{x}+\psi+I w\right)^{2}+k_{3}\left(w_{x}-I \varphi\right)^{2}\right) \mathrm{d} x \\
& -\int_{0}^{L}\left[\left(\int_{0}^{+\infty} g_{1}(s) \mathrm{d} s\right) \varphi_{x}^{2}+\left(\int_{0}^{+\infty} g_{2}(s) \mathrm{d} s\right) \psi_{x}^{2}\right. \\
& \left.+\left(\int_{0}^{+\infty} g_{3}(s) \mathrm{d} s\right) w_{x}^{2}\right] \mathrm{d} x .
\end{aligned}
$$

Remark 1 It is easy to check that there exists a positive constant $\bar{k}_{0}$ such that, for any $(\varphi, \psi, w) \in$ $H_{0}^{1}(0, L) \times H_{0}^{1}(0, L) \times H_{0}^{1}(0, L)$,

$$
\bar{k}_{0} \int_{0}^{L}\left(\varphi_{x}^{2}+\psi_{x}^{2}+w_{x}^{2}\right) \mathrm{d} x \leq \int_{0}^{L}\left(k_{2} \psi_{x}^{2}+k_{1}\left(\varphi_{x}+\psi+I w\right)^{2}+k_{3}\left(w_{x}-I \varphi\right)^{2}\right) \mathrm{d} x .
$$

Therefore, let

$$
g_{i}^{0}:=\int_{0}^{+\infty} g_{i}(s) \mathrm{d} s<\bar{k}_{0}, \quad i=1,2,3
$$

then (2.5) is satisfied with

$$
k_{0}=\bar{k}_{0}-\max \left\{g_{1}^{0}, g_{2}^{0}, g_{3}^{0}\right\} .
$$

On the other hand, due to Poincaré's inequality, there exists a positive constant $\tilde{k}_{0}$ such that, for $(\varphi, \psi, w) \in H_{0}^{1}(0, L) \times H_{0}^{1}(0, L) \times H_{0}^{1}(0, L)$,

$$
\int_{0}^{L}\left(k_{2} \psi_{x}^{2}+k_{1}\left(\varphi_{x}+\psi+I w\right)^{2}+k_{3}\left(w_{x}-I \varphi\right)^{2}\right) \mathrm{d} x \leq \tilde{k}_{0} \int_{0}^{L}\left(\varphi_{x}^{2}+\psi_{x}^{2}+w_{x}^{2}\right) \mathrm{d} x .
$$

Remark 2 Under hypothesis (G1), $H_{i}^{*}$ and $\mathscr{H}$ are Hilbert spaces, respectively, with the inner products that generate the norms

$$
\left\|\eta_{i}\right\|_{H_{i}^{*}}^{2}=\int_{0}^{L} \int_{0}^{+\infty} g_{i}(s)\left(\partial_{x} \eta_{i}\right)^{2} \mathrm{~d} s \mathrm{~d} x
$$

and

$$
\|U\|_{\mathscr{H}}^{2}=\int_{0}^{L}\left(\rho_{1} \varphi_{t}^{2}+\rho_{2} \psi_{t}^{2}+\rho_{1} w_{t}^{2}+k_{2} \psi_{x}^{2}+k_{1}\left(\varphi_{x}+\psi+I w\right)^{2}+k_{3}\left(w_{x}-I \varphi\right)^{2}+\rho_{3} \theta^{2}\right.
$$




$$
\begin{aligned}
& \left.+\frac{\tau}{\gamma} q^{2}\right) \mathrm{d} x-\int_{0}^{L}\left(g_{1}^{0} \varphi_{x}^{2}+g_{2}^{0} \psi_{x}^{2}+g_{3}^{0} w_{x}^{2}\right) \mathrm{d} x+\sum_{i=1}^{3} \zeta_{i} \tau_{i}(t) \int_{0}^{L} \int_{0}^{1} z_{i}^{2} \mathrm{~d} \rho \mathrm{d} x \\
& +\left\|\eta_{1}\right\|_{H_{1}^{*}}^{2}+\left\|\eta_{2}\right\|_{H_{2}^{*}}^{2}+\left\|\eta_{3}\right\|_{H_{3}^{*}}^{2},
\end{aligned}
$$

where $\zeta_{i}(i=1,2,3)$ are positive constants such that

$$
\left\{\begin{array}{l}
\frac{\left|\mu_{2}\right|}{\sqrt{1-d_{1}}} \leq \zeta_{1} \leq 2 \mu_{1}-\frac{\left|\mu_{2}\right|}{\sqrt{1-d_{1}}} \\
\frac{\left|\tilde{\mu}_{2}\right|}{\sqrt{1-d_{2}}} \leq \zeta_{2} \leq 2 \tilde{\mu}_{1}-\frac{\left|\tilde{\mu}_{2}\right|}{\sqrt{1-d_{2}}} \\
\frac{\left|\tilde{\tilde{\mu}}_{2}\right|}{\sqrt{1-d_{3}}} \leq \zeta_{3} \leq 2 \tilde{\tilde{\mu}}_{1}-\frac{\left|\tilde{\tilde{\mu}}_{2}\right|}{\sqrt{1-d_{3}}}
\end{array}\right.
$$

(G2) For $i=1,2,3$, there exist positive constants $\delta_{i}(i=1,2,3)$ such that

$$
g_{i}^{\prime}(s) \leq-\delta_{i} g_{i}(s), \quad \forall s \in \mathbb{R}^{+}
$$

or there exists an increasing strictly convex function $G: \mathbb{R}^{+} \rightarrow \mathbb{R}^{+}$of class $C^{1}\left(\mathbb{R}^{+}\right) \cap C^{2}(0,+\infty)$ satisfying

$$
G(0)=G^{\prime}(0)=0 \quad \text { and } \quad \lim _{t \rightarrow+\infty} G^{\prime}(t)=+\infty
$$

such that

$$
\int_{0}^{+\infty} \frac{g_{i}(s)}{G^{-1}\left(-g_{i}^{\prime}(s)\right)} \mathrm{d} s+\sup _{s \in \mathbb{R}^{+}} \frac{g_{i}(s)}{G^{-1}\left(-g_{i}^{\prime}(s)\right)}<+\infty .
$$

Now, we state the well-posedness result of problem (2.9)

Theorem 2.1 Assume that $(G 1)$ holds. Let $U^{0} \in \mathscr{H}$, then problem (2.9) has a unique weak solution

$$
U \in C\left(\mathbb{R}^{+} ; \mathscr{H}\right)
$$

Moreover, if $U^{0} \in D(\mathscr{A})$, then

$$
U \in C\left(\mathbb{R}^{+} ; D(\mathscr{A})\right) \cap C^{1}\left(\mathbb{R}^{+} ; \mathscr{H}\right) .
$$

The energy associated with problem (2.9) is defined by

$$
\begin{aligned}
E(t)= & \frac{1}{2}\left(\int _ { 0 } ^ { L } \left(\rho_{1} \varphi_{t}^{2}+\rho_{2} \psi_{t}^{2}+\rho_{1} w_{t}^{2}+k_{2} \psi_{x}^{2}+k_{1}\left(\varphi_{x}+\psi+I w\right)^{2}+k_{3}\left(w_{x}-I \varphi\right)^{2}+\rho_{3} \theta^{2}\right.\right. \\
& \left.+\frac{\tau}{\gamma} q^{2}\right) \mathrm{d} x-\int_{0}^{L}\left(g_{1}^{0} \varphi_{x}^{2}+g_{2}^{0} \psi_{x}^{2}+g_{3}^{0} w_{x}^{2}\right) \mathrm{d} x+\sum_{i=1}^{3} \zeta_{i} \tau_{i}(t) \int_{0}^{L} \int_{0}^{1} z_{i}^{2} \mathrm{~d} \rho \mathrm{d} x \\
& \left.+\left\|\eta_{1}\right\|_{H_{1}^{*}}^{2}+\left\|\eta_{2}\right\|_{H_{2}^{*}}^{2}+\left\|\eta_{3}\right\|_{H_{3}^{*}}^{2}\right) .
\end{aligned}
$$

Our decay results read as follows:

Theorem 2.2 Let $U^{0} \in \mathscr{H}$ be given. Assume that $(G 1)$ and $(G 2)$ hold,

(i) If for all $i=1,2,3,(2.18)$ holds, then there exist positive constants $c^{\prime}, c^{\prime \prime}$ such that

$$
E(t) \leq c^{\prime \prime} e^{-c^{\prime} t}
$$


(ii) If for $i=1,2,3$, either (2.18) holds or (2.19), (2.20) and

$$
\exists M_{i} \geq 0: \int_{0}^{L}\left(\partial_{x} \eta_{i}^{0}\right)^{2} \mathrm{~d} x \leq M_{i}, \forall s>0 \text { hold, }
$$

then there exist positive constants $c^{\prime}, c^{\prime \prime}$ and $\epsilon_{0}$ such that

$$
E(t) \leq c^{\prime \prime} H^{-1}\left(c^{\prime} t\right)
$$

where

$$
H(s)=\int_{s}^{1} \frac{1}{\tau G^{\prime}\left(\epsilon_{0} \tau\right)} \mathrm{d} \tau, \forall s \in(0,1]
$$

\section{Proof of the well-posedness}

In this section, we prove the well-posedness of the solution of problem (2.11). For this purpose, we will follow the method used in [4],[14] and [23] with the necessary modification imposed by the nature of our problem.

Proof of Theorem 2.1. In order to prove result stated in Theorem 2.1, first, we prove that the operator $\mathscr{A}$ is dissipative. A simple computation implies that, for any $U \in D(\mathscr{A})$,

$$
\begin{aligned}
\langle\mathscr{A} U, U\rangle_{\mathscr{H}}= & -\left(\mu_{1}-\frac{\zeta_{1}}{2}\right) \int_{0}^{L} \varphi_{t}^{2} \mathrm{~d} x-\left(\tilde{\mu}_{1}-\frac{\zeta_{2}}{2}\right) \int_{0}^{L} \psi_{t}^{2} \mathrm{~d} x-\left(\tilde{\tilde{\mu}}_{1}-\frac{\zeta_{3}}{2}\right) \int_{0}^{L} w_{t}^{2} \mathrm{~d} x \\
& -\mu_{2} \int_{0}^{L} z_{1}(x, 1, t) \varphi_{t} \mathrm{~d} x-\tilde{\mu}_{2} \int_{0}^{L} z_{2}(x, 1, t) \psi_{t} \mathrm{~d} x-\tilde{\tilde{\mu}}_{2} \int_{0}^{L} z_{3}(x, 1, t) w_{t} \mathrm{~d} x \\
& -\frac{1}{2} \int_{0}^{L} \int_{0}^{+\infty} g_{1}(s) \partial_{s}\left(\partial_{x} \eta_{1}\right)^{2} \mathrm{~d} s \mathrm{~d} x-\frac{1}{2} \int_{0}^{L} \int_{0}^{+\infty} g_{2}(s) \partial_{s}\left(\partial_{x} \eta_{2}\right)^{2} \mathrm{~d} s \mathrm{~d} x \\
& -\frac{1}{2} \int_{0}^{L} \int_{0}^{+\infty} g_{3}(s) \partial_{s}\left(\partial_{x} \eta_{3}\right)^{2} \mathrm{~d} s \mathrm{~d} x-\int_{0}^{L} \frac{1}{\gamma} q^{2} \mathrm{~d} x \\
& -\sum_{i=1}^{3} \frac{\zeta_{i}\left(1-\tau_{i}^{\prime}(t)\right)}{2} \int_{0}^{L} z_{i}^{2}(x, 1, t) \mathrm{d} x .
\end{aligned}
$$

Integrating by parts, using (G1) and the boundary conditions in (2.10), we get

$$
\begin{aligned}
\langle\mathscr{A} U, U\rangle_{\mathscr{H}}= & -\left(\mu_{1}-\frac{\zeta_{1}}{2}\right) \int_{0}^{L} \varphi_{t}^{2} \mathrm{~d} x-\left(\tilde{\mu}_{1}-\frac{\zeta_{2}}{2}\right) \int_{0}^{L} \psi_{t}^{2} \mathrm{~d} x-\left(\tilde{\tilde{\mu}}_{1}-\frac{\zeta_{3}}{2}\right) \int_{0}^{L} w_{t}^{2} \mathrm{~d} x \\
& -\mu_{2} \int_{0}^{L} z_{1}(x, 1, t) \varphi_{t} \mathrm{~d} x-\tilde{\mu}_{2} \int_{0}^{L} z_{2}(x, 1, t) \psi_{t} \mathrm{~d} x-\tilde{\tilde{\mu}}_{2} \int_{0}^{L} z_{3}(x, 1, t) w_{t} \mathrm{~d} x \\
& +\frac{1}{2} \int_{0}^{L} \int_{0}^{+\infty}\left(g_{1}^{\prime}(s)\left(\partial_{x} \eta_{1}\right)^{2}+g_{2}^{\prime}(s)\left(\partial_{x} \eta_{2}\right)^{2}+g_{3}^{\prime}(s)\left(\partial_{x} \eta_{3}\right)^{2}\right) \mathrm{d} s \mathrm{~d} x-\int_{0}^{L} \frac{1}{\gamma} q^{2} \mathrm{~d} x \\
& -\sum_{i=1}^{3} \frac{\zeta_{i}\left(1-\tau_{i}^{\prime}(t)\right)}{2} \int_{0}^{L} z_{i}^{2}(x, 1, t) \mathrm{d} x .
\end{aligned}
$$

Then, using Young's inequality, we obtain

$$
\langle\mathscr{A} U, U\rangle_{\mathscr{H}} \leq-\left(\mu_{1}-\frac{\zeta_{1}}{2}-\frac{\left|\mu_{2}\right|}{2 \sqrt{1-d}}\right) \int_{0}^{L} \varphi_{t}^{2} \mathrm{~d} x-\left(\tilde{\mu}_{1}-\frac{\zeta_{2}}{2}-\frac{\left|\tilde{\mu}_{2}\right|}{2 \sqrt{1-d}}\right) \int_{0}^{L} \psi_{t}^{2} \mathrm{~d} x
$$




$$
\begin{aligned}
& -\left(\tilde{\tilde{\mu}}_{1}-\frac{\zeta_{3}}{2}-\frac{\left|\tilde{\tilde{\mu}}_{2}\right|}{2 \sqrt{1-d}}\right) \int_{0}^{L} w_{t}^{2} \mathrm{~d} x \\
& -\left(\frac{\zeta_{1}\left(1-\tau_{1}^{\prime}(t)\right)}{2}-\frac{\left|\mu_{2}\right| \sqrt{1-d}}{2}\right) \int_{0}^{L} z_{1}^{2}(x, 1, t) \mathrm{d} x \\
& -\left(\frac{\zeta_{2}\left(1-\tau_{2}^{\prime}(t)\right)}{2}-\frac{\left|\tilde{\mu}_{2}\right| \sqrt{1-d}}{2}\right) \int_{0}^{L} z_{2}^{2}(x, 1, t) \mathrm{d} x \\
& -\left(\frac{\zeta_{3}\left(1-\tau_{3}^{\prime}(t)\right)}{2}-\frac{\left|\tilde{\tilde{\mu}}_{2}\right| \sqrt{1-d}}{2}\right) \int_{0}^{L} z_{3}^{2}(x, 1, t) \mathrm{d} x \\
& +\frac{1}{2} \int_{0}^{L} \int_{0}^{+\infty}\left(g_{1}^{\prime}(s)\left(\partial_{x} \eta_{1}\right)^{2}+g_{2}^{\prime}(s)\left(\partial_{x} \eta_{2}\right)^{2}+g_{3}^{\prime}(s)\left(\partial_{x} \eta_{3}\right)^{2}\right) \mathrm{d} s \mathrm{~d} x-\int_{0}^{L} \frac{1}{\gamma} q^{2} \mathrm{~d} x .
\end{aligned}
$$

Notice the fact that, for $i=1,2,3$, the kernel $g_{i}$ is non-increasing and using (2.3), (2.17), we obtain

$$
\langle\mathscr{A} U, U\rangle_{\mathscr{H}} \leq 0
$$

Hence, $\mathscr{A}$ is a dissipative operator.

Next, we prove that the operator $I d-\mathscr{A}$ is surjective. For this purpose, Given

$$
F=\left(f_{1}, f_{2}, f_{3}, f_{4}, f_{5}, f_{6}, f_{7}, f_{8}, f_{9}, f_{10}, f_{11}, f_{12}, f_{13}, f_{14}\right) \in \mathscr{H}
$$

we prove that there exists

$$
V=\left(v_{1}, v_{2}, v_{3}, v_{4}, v_{5}, v_{6}, v_{7}, v_{8}, z_{1}, z_{2}, z_{3}, v_{9}, v_{10}, v_{11}\right) \in D(\mathscr{A})
$$

satisfying

$$
(I d-\mathscr{A}) V=F
$$


Equation (3.3) is equivalent to

$$
\begin{aligned}
& \left\{\begin{array}{l}
v_{1}-v_{6}=f_{1}, \\
v_{2}-v_{7}=f_{2}, \\
v_{3}-v_{8}=f_{3},
\end{array}\right. \\
& v_{3}-v_{8}=f_{3} \text {, } \\
& \rho_{3} v_{4}+\partial_{x} v_{5}+\sigma \partial_{x} v_{6}=\rho_{3} f_{4} \\
& (1+\tau) v_{5}+\gamma \partial_{x} v_{4}=\tau f_{5}, \\
& \rho_{1} v_{6}-\left(k_{1}-\int_{0}^{+\infty} g_{1}(s) \mathrm{d} s\right) \partial_{x x} v_{1}+I^{2} k_{3} v_{1}-k_{1} \partial_{x} v_{2}-I\left(k_{1}+k_{3}\right) \partial_{x} v_{3}+\sigma \partial_{x} v_{4} \\
& +\mu_{1} v_{6}+\mu_{2} z_{1}(., 1)-\int_{0}^{+\infty} g_{1}(s) \partial_{x x} v_{9} \mathrm{~d} s=\rho_{1} f_{6} \\
& \rho_{2} v_{7}+k_{1} \partial_{x} v_{1}-\left(k_{2}-\int_{0}^{+\infty} g_{2}(s) \mathrm{d} s\right) \partial_{x x} v_{2}+k_{1} v_{2}+I k_{1} v_{3} \\
& +\tilde{\mu}_{1} v_{7}+\tilde{\mu}_{2} z_{2}(., 1)-\int_{0}^{+\infty} g_{2}(s) \partial_{x x} v_{10} \mathrm{~d} s=\rho_{2} f_{7}, \\
& \rho_{1} v_{8}+I\left(k_{1}+k_{3}\right) \partial_{x} v_{1}+I k_{1} v_{2}-\left(k_{3}-\int_{0}^{\infty} g_{3}(s) \mathrm{d} s\right) \partial_{x x} v_{3}+I^{2} k_{1} v_{3} \\
& +\tilde{\tilde{\mu}}_{1} v_{8}+\tilde{\tilde{\mu}}_{2} z_{3}(., 1)-\int_{0}^{\infty} g_{3}(s) \partial_{x x} v_{11} \mathrm{~d} s=\rho_{1} f_{8}, \\
& z_{1}+\frac{1-\tau_{1}^{\prime}(t) \rho}{\tau_{1}(t)} z_{1 \rho}=f_{9} \\
& z_{2}+\frac{1-\tau_{2}^{\prime}(t) \rho}{\tau_{2}(t)} z_{1 \rho}=f_{10} \\
& z_{3}+\frac{1-\tau_{3}^{\prime}(t) \rho}{\tau_{3}(t)} z_{1 \rho}=f_{11} \\
& v_{9}-v_{6}+\partial_{s} v_{9}=f_{12}, \\
& v_{10}-v_{7}+\partial_{s} v_{10}=f_{13} \\
& v_{11}-v_{8}+\partial_{s} v_{11}=f_{14} \text {. }
\end{aligned}
$$

Suppose $v_{1}, v_{2}, v_{3}, v_{5}$ are found with the appropriate regularity, then $(3.4)_{1}-(3.4)_{3}$ and $(3.4)_{5}$ give

$$
\begin{array}{r}
v_{6}=v_{1}-f_{1} \in H_{0}^{1}(0, L), \\
v_{7}=v_{2}-f_{2} \in H_{0}^{1}(0, L), \\
v_{8}=v_{3}-f_{3} \in H_{0}^{1}(0, L), \\
\partial_{x} v_{4}=-\frac{(1+\tau)}{\gamma} v_{5}+\frac{\tau}{\gamma} f_{5} \in L_{*}^{2}(0, L) .
\end{array}
$$

The last equation of (3.5) yield

$$
v_{4}=-\frac{(1+\tau)}{\gamma} \int_{0}^{x} v_{5} \mathrm{~d} y+\frac{\tau}{\gamma} \int_{0}^{x} f_{5} \mathrm{~d} y
$$

then

$$
v_{4}(0, t)=v_{4}(L, t)=0
$$


On the other hand, by using (2.6), we can find $z_{1}, z_{2}, z_{3}$ as

$$
z_{1}(x, 0)=v_{6}, z_{2}(x, 0)=v_{7}, z_{3}(x, 0)=v_{8}, \text { for } x \in(0, L) .
$$

Following the same approach in [23] and using $(3.4)_{9^{-}}(3.4)_{11}$, we obtain

$$
z_{1}(x, \rho)=v_{6} e^{-\rho \tau_{1}(t)}+\tau_{1}(t) e^{-\rho \tau_{1}(t)} \int_{0}^{\rho} f_{9}(x, \sigma) e^{\sigma \tau_{1}(t)} \mathrm{d} \sigma, \text { if } \tau_{1}^{\prime}(t)=0
$$

and

$$
z_{1}(x, \rho)=v_{6} e^{\vartheta_{1 \rho}(t)}+e^{\vartheta_{1 \rho}(t)} \int_{0}^{\rho} \frac{f_{9}(x, \sigma) \tau_{1}(t)}{1-\tau_{1}^{\prime}(t) \sigma} e^{-\vartheta_{1 \sigma}(t)} \mathrm{d} \sigma, \text { if } \tau_{1}^{\prime}(t) \neq 0,
$$

Hence, from (3.5), we obtain

$$
z_{1}(x, \rho)=v_{1} e^{-\rho \tau_{1}(t)}-f_{1} e^{-\rho \tau_{1}(t)}+\tau_{1}(t) e^{-\rho \tau_{1}(t)} \int_{0}^{\rho} f_{9}(x, \sigma) e^{\sigma \tau_{1}(t)} \mathrm{d} \sigma, \text { if } \tau_{1}^{\prime}(t)=0
$$

and

$$
z_{1}(x, \rho)=v_{1} e^{\vartheta_{1 \rho}(t)}-f_{1} e^{\vartheta_{1 \rho}(t)}+e^{\vartheta_{1 \rho}(t)} \int_{0}^{\rho} \frac{f_{9}(x, \sigma) \tau_{1}(t)}{1-\tau_{1}^{\prime}(t) \sigma} e^{-\vartheta_{1 \sigma}(t)} \mathrm{d} \sigma, \text { if } \tau_{1}^{\prime}(t) \neq 0 .
$$

Similarly, we get

$$
\begin{aligned}
& z_{2}(x, \rho)=v_{2} e^{-\rho \tau_{2}(t)}-f_{2} e^{-\rho \tau_{2}(t)}+\tau_{2}(t) e^{-\rho \tau_{2}(t)} \int_{0}^{\rho} f_{10}(x, \sigma) e^{\sigma \tau_{2}(t)} \mathrm{d} \sigma, \text { if } \tau_{2}^{\prime}(t)=0, \\
& z_{2}(x, \rho)=v_{2} e^{\vartheta_{2 \rho}(t)}-f_{2} e^{\vartheta_{2 \rho}(t)}+e^{\vartheta_{2 \rho}(t)} \int_{0}^{\rho} \frac{f_{10}(x, \sigma) \tau_{2}(t)}{1-\tau_{2}^{\prime}(t) \sigma} e^{-\vartheta_{2 \sigma}(t)} \mathrm{d} \sigma, \text { if } \tau_{2}^{\prime}(t) \neq 0 . \\
& z_{3}(x, \rho)=v_{3} e^{-\rho \tau_{3}(t)}-f_{3} e^{-\rho \tau_{3}(t)}+\tau_{3}(t) e^{-\rho \tau_{3}(t)} \int_{0}^{\rho} f_{11}(x, \sigma) e^{\sigma \tau_{3}(t)} \mathrm{d} \sigma, \text { if } \tau_{3}^{\prime}(t)=0
\end{aligned}
$$

and

$$
z_{3}(x, \rho)=v_{3} e^{\vartheta_{3 \rho}(t)}-f_{3} e^{\vartheta_{3 \rho}(t)}+e^{\vartheta_{3 \rho}(t)} \int_{0}^{\rho} \frac{f_{11}(x, \sigma) \tau_{3}(t)}{1-\tau_{3}^{\prime}(t) \sigma} e^{-\vartheta_{3 \sigma}(t)} \mathrm{d} \sigma, \text { if } \tau_{3}^{\prime}(t) \neq 0,
$$

where

$$
\vartheta_{i \rho}(t)=\frac{\tau_{i}(t)}{\tau_{i}^{\prime}(t)} \ln \left(1-\tau_{i}^{\prime}(t) \rho\right), i=1,2,3
$$

From (3.8)-(3.13), we have

$$
\begin{aligned}
& z_{1}(x, 1)=\left\{\begin{array}{l}
v_{1} e^{-\tau_{1}(t)}+z_{10}(x), \text { if } \tau_{1}^{\prime}(t)=0, \\
v_{1} e^{\vartheta_{1 \rho}(t)}+z_{10}(x), \text { if } \tau_{1}^{\prime}(t) \neq 0 .
\end{array}\right. \\
& z_{2}(x, 1)=\left\{\begin{array}{l}
v_{2} e^{-\tau_{2}(t)}+z_{20}(x), \text { if } \tau_{2}^{\prime}(t)=0, \\
v_{2} e^{\vartheta_{2 \rho}(t)}+z_{20}(x), \text { if } \tau_{2}^{\prime}(t) \neq 0 .
\end{array}\right. \\
& z_{3}(x, 1)=\left\{\begin{array}{l}
v_{3} e^{-\tau_{3}(t)}+z_{30}(x), \text { if } \tau_{3}^{\prime}(t)=0, \\
v_{3} e^{\vartheta_{3 \rho}(t)}+z_{30}(x), \text { if } \tau_{3}^{\prime}(t) \neq 0,
\end{array}\right.
\end{aligned}
$$


where

$$
\begin{gathered}
z_{10}(x)=\left\{\begin{array}{l}
-f_{1} e^{-\tau_{1}(t)}+\tau_{1}(t) e^{-\tau_{1}(t)} \int_{0}^{1} f_{9}(x, \sigma) e^{\sigma \tau_{1}(t)} \mathrm{d} \sigma, \text { if } \tau_{1}^{\prime}(t)=0, \\
-f_{1} e^{\vartheta_{1 \rho}(t)}+e^{\vartheta_{1 \rho}(t)} \int_{0}^{\rho} \frac{f_{9}(x, \sigma) \tau_{1}(t)}{1-\tau_{1}^{\prime}(t) \sigma} e^{-\vartheta_{1 \sigma}(t)} \mathrm{d} \sigma, \text { if } \tau_{1}^{\prime}(t) \neq 0 .
\end{array}\right. \\
z_{20}(x)=\left\{\begin{array}{l}
-f_{2} e^{-\tau_{2}(t)}+\tau_{2}(t) e^{-\tau_{2}(t)} \int_{0}^{1} f_{10}(x, \sigma) e^{\sigma \tau_{2}(t)} \mathrm{d} \sigma, \text { if } \tau_{2}^{\prime}(t)=0, \\
-f_{2} e^{\vartheta_{2 \rho}(t)}+e^{\vartheta_{2 \rho}(t)} \int_{0}^{\rho} \frac{f_{10}(x, \sigma) \tau_{2}(t)}{1-\tau_{2}^{\prime}(t) \sigma} e^{-\vartheta_{2 \sigma}(t)} \mathrm{d} \sigma, \text { if } \tau_{2}^{\prime}(t) \neq 0 .
\end{array}\right. \\
z_{30}(x)=\left\{\begin{array}{l}
-f_{3} e^{-\tau_{3}(t)}+\tau_{3}(t) e^{-\tau_{3}(t)} \int_{0}^{1} f_{11}(x, \sigma) e^{\sigma \tau_{3}(t)} \mathrm{d} \sigma, \text { if } \tau_{3}^{\prime}(t)=0, \\
-f_{3} e^{\vartheta_{3 \rho}(t)}+e^{\vartheta_{3 \rho}(t)} \int_{0}^{\rho} \frac{f_{11}(x, \sigma) \tau_{3}(t)}{1-\tau_{3}^{\prime}(t) \sigma} e^{-\vartheta_{3 \sigma}(t)} \mathrm{d} \sigma, \text { if } \tau_{3}^{\prime}(t) \neq 0 .
\end{array}\right.
\end{gathered}
$$

It is clear from the above formula that $z_{10}, z_{20}$ and $z_{30}$ depend only on $f_{i}, i=1,2,3,9,10,11$.

Next, (3.4) $)_{12}-(3.4)_{14}$ and (3.5) imply

$$
\partial_{s} v_{9}+v_{9}=v_{1}-f_{1}+f_{12}, \partial_{s} v_{10}+v_{10}=v_{2}-f_{2}+f_{13}, \partial_{s} v_{11}+v_{11}=v_{3}-f_{3}+f_{14} .
$$

By solving above three differential equations and noticing that $v_{9}(0)=v_{10}(0)=v_{11}(0)=0$, we get

$$
\begin{gathered}
v_{9}=\left(1-e^{-s}\right)\left(v_{1}-f_{1}\right)+\int_{0}^{s} e^{\tau-s} f_{12}(\tau) \mathrm{d} \tau \in H_{1}^{*} . \\
v_{10}=\left(1-e^{-s}\right)\left(v_{2}-f_{2}\right)+\int_{0}^{s} e^{\tau-s} f_{13}(\tau) \mathrm{d} \tau \in H_{2}^{*}, \\
v_{11}=\left(1-e^{-s}\right)\left(v_{3}-f_{3}\right)+\int_{0}^{s} e^{\tau-s} f_{14}(\tau) \mathrm{d} \tau \in H_{3}^{*} .
\end{gathered}
$$


By using (3.5)-(3.6) and (3.20), it can be shown that $v_{1}, v_{2}, v_{3}$ and $v_{5}$ satisfy

$$
\left\{\begin{array}{l}
\left(\rho_{1}+\mu_{1}\right) v_{1}-\left(k_{1}-\int_{0}^{+\infty} e^{-s} g_{1}(s) \mathrm{d} s\right) \partial_{x x} v_{1}+I^{2} k_{3} v_{1}-k_{1} \partial_{x} v_{2}-I\left(k_{1}+k_{3}\right) \partial_{x} v_{3} \\
-\frac{\sigma(1+\tau)}{\gamma} v_{5}+\mu_{2} z_{1}(\cdot, 1)=\rho_{1}\left(f_{1}+f_{6}\right)+\mu_{1} f_{1}-\int_{0}^{+\infty} g_{1}(s)\left(1-e^{-s}\right) \partial_{x x} f_{1} \mathrm{~d} s \\
-\frac{\tau \sigma}{\gamma} f_{5}+\int_{0}^{+\infty} g_{1}(s) \partial_{x x}\left(\int_{0}^{s} e^{\tau-s} f_{12}(\tau) \mathrm{d} \tau\right) \mathrm{d} s \\
\left(\rho_{2}+\tilde{\mu}_{1}\right) v_{2}+k_{1} \partial_{x} v_{1}-\left(k_{2}-\int_{0}^{+\infty} e^{-s} g_{2}(s) \mathrm{d} s\right) \partial_{x x} v_{2}+k_{1} v_{2}+I k_{1} v_{3}+\tilde{\mu}_{2} z_{2}(\cdot, 1) \\
=\rho_{2}\left(f_{2}+f_{7}\right)+\tilde{\mu}_{1} f_{2}-\int_{0}^{+\infty} g_{2}(s)\left(1-e^{-s}\right) \partial_{x x} f_{2} \mathrm{~d} s+\int_{0}^{+\infty} g_{2}(s) \partial_{x x}\left(\int_{0}^{s} e^{\tau-s} f_{13}(\tau) \mathrm{d} \tau\right) \mathrm{d} s \\
\left(\rho_{1}+\tilde{\tilde{\mu}}_{1}\right) v_{3}+I\left(k_{1}+k_{3}\right) \partial_{x} v_{1}+I k_{1} v_{2}-\left(k_{3}-\int_{0}^{\infty} e^{-s} g_{3}(s) \mathrm{d} s\right) \partial_{x x} v_{3}+I^{2} k_{1} v_{3}+\tilde{\mu}_{2} z_{3}(\cdot, 1) \\
=\rho_{1}\left(f_{3}+f_{8}\right)+\tilde{\tilde{\mu}}_{1} f_{3}-\int_{0}^{+\infty} g_{3}(s)\left(1-e^{-s}\right) \partial_{x x} f_{3} \mathrm{~d} s+\int_{0}^{+\infty} g_{3}(s) \partial_{x x}\left(\int_{0}^{s} e^{\tau-s} f_{14}(\tau) \mathrm{d} \tau\right) \mathrm{d} s \\
\frac{(1+\tau)}{\gamma} \rho_{3} \int_{0}^{x} v_{5} \mathrm{~d} y-\partial_{x} v_{5}-\sigma \partial_{x} v_{1}=\frac{\tau \rho_{3}}{\gamma} \int_{0}^{x} f_{5} \mathrm{~d} y-\sigma \partial_{x} f_{1}-\rho_{3} f_{4} \cdot
\end{array}\right.
$$

Then, multiplying $(3.21)_{1}-(3.21)_{4}$ by $\tilde{v}_{1}, \tilde{v}_{2}, \tilde{v}_{3}$ and $\frac{1+\tau}{\gamma} \int_{0}^{x} \tilde{v}_{5}$, respectively, integrating their sum over $(0, L)$ and using (3.14)-(3.16), we get

$$
\begin{array}{r}
a\left(\left(v_{1}, v_{2}, v_{3}, v_{5}\right)^{T},\left(\tilde{v}_{1}, \tilde{v}_{2}, \tilde{v}_{3}, \tilde{v}_{5}\right)^{T}\right)=\tilde{a}\left(\left(\tilde{v}_{1}, \tilde{v}_{2}, \tilde{v}_{3}, \tilde{v}_{5}\right)^{T}\right), \\
\forall\left(\tilde{v}_{1}, \tilde{v}_{2}, \tilde{v}_{3}, \tilde{v}_{5}\right)^{T} \in H_{0}^{1}(0, L) \times H_{0}^{1}(0, L) \times H_{0}^{1}(0, L) \times L_{*}^{2}(0, L),
\end{array}
$$

where

$$
\begin{aligned}
& a\left(\left(v_{1}, v_{2}, v_{3}, v_{5}\right)^{T},\left(\tilde{v}_{1}, \tilde{v}_{2}, \tilde{v}_{3}, \tilde{v}_{5}\right)^{T}\right) \\
& =\int_{0}^{L}\left(k_{1}\left(\partial_{x} v_{1}+v_{2}+I v_{3}\right)\left(\partial_{x} \tilde{v}_{1}+\tilde{v}_{2}+I \tilde{v}_{3}\right) \mathrm{d} x+\int_{0}^{L} k_{3}\left(\partial_{x} v_{3}-I v_{1}\right)\left(\partial_{x} \tilde{v}_{3}-I \tilde{v}_{1}\right) \mathrm{d} x\right. \\
& +\int_{0}^{L} k_{2} \partial_{x} v_{2} \partial_{x} \tilde{v}_{2} \mathrm{~d} x+\int_{0}^{L}\left(\left(\rho_{1}+\mu_{1}+e^{-\tau_{1}(t)}\right) v_{1} \tilde{v}_{1}+\left(\rho_{2}+\tilde{\mu}_{1}+e^{-\tau_{2}(t)}\right) v_{2} \tilde{v}_{2}\right. \\
& \left.+\left(\rho_{1}+\tilde{\tilde{\mu}}_{1}+e^{-\tau_{1}(t)}\right) v_{3} \tilde{v}_{3}+\frac{1+\tau}{\gamma} v_{5} \tilde{v}_{5}\right) \mathrm{d} x+\frac{\rho_{3}(1+\tau)^{2}}{\gamma^{2}} \int_{0}^{L}\left(\int_{0}^{x} v_{5} \mathrm{~d} y \int_{0}^{x} \tilde{v}_{5} \mathrm{~d} y\right) \mathrm{d} x \\
& +\int_{0}^{L}\left(-\tilde{g}_{1}^{0} \partial_{x} v_{1} \partial_{x} \tilde{v}_{1}-\tilde{g}_{2}^{0} \partial_{x} v_{2} \partial_{x} \tilde{v}_{2}-\tilde{g}_{3}^{0} \partial_{x} v_{3} \partial_{x} \tilde{v}_{3}\right) \mathrm{d} x+\frac{\sigma(1+\tau)}{\gamma} \int_{0}^{L} v_{1} \tilde{v}_{5} \mathrm{~d} x \\
& -\frac{\sigma(1+\tau)}{\gamma} \int_{0}^{L} v_{5} \tilde{v}_{1} \mathrm{~d} x, \quad \tilde{g}_{1}^{0}=\int_{0}^{+\infty} e^{-s} g_{i}(s) \mathrm{d} s
\end{aligned}
$$

and

$$
\tilde{a}\left(\left(\tilde{v}_{1}, \tilde{v}_{2}, \tilde{v}_{3}, \tilde{v}_{5}\right)^{T}\right)=\int_{0}^{L}\left(\rho_{1}\left(f_{1}+f_{6}\right) \tilde{v}_{1}+\rho_{2}\left(f_{2}+f_{7}\right) \tilde{v}_{2}+\rho_{1}\left(f_{3}+f_{8}\right) \tilde{v}_{3}\right) \mathrm{d} x
$$




$$
\begin{aligned}
& +\int_{0}^{L}\left(\left(g_{1}^{0}-\tilde{g}_{1}^{0}\right) \partial_{x} f_{1} \partial_{x} \tilde{v}_{1}+\left(g_{2}^{0}-\tilde{g}_{2}^{0}\right) \partial_{x} f_{2} \partial_{x} \tilde{v}_{2}+\left(g_{3}^{0}-\tilde{g}_{3}^{0}\right) \partial_{x} f_{3} \partial_{x} \tilde{v}_{3}\right) \mathrm{d} x \\
& -\int_{0}^{L}\left(\int_{0}^{+\infty} g_{1}(s) \int_{0}^{s} e^{\tau-s} \partial_{x} f_{9}(\tau) \mathrm{d} \tau \mathrm{d} s\right) \partial_{x} \tilde{v}_{1} \mathrm{~d} x+\frac{\tau \sigma}{\gamma} \int_{0}^{L} f_{5} \tilde{v}_{1} \mathrm{~d} x \\
& -\int_{0}^{L}\left(\int_{0}^{+\infty} g_{2}(s) \int_{0}^{s} e^{\tau-s} \partial_{x} f_{10}(\tau) \mathrm{d} \tau \mathrm{d} s\right) \partial_{x} \tilde{v}_{2} \mathrm{~d} x+\frac{\sigma(1+\tau)}{\gamma} \int_{0}^{L} f_{1} \tilde{v}_{5} \mathrm{~d} x \\
& -\int_{0}^{L}\left(\int_{0}^{+\infty} g_{3}(s) \int_{0}^{s} e^{\tau-s} \partial_{x} f_{11}(\tau) \mathrm{d} \tau \mathrm{d} s\right) \partial_{x} \tilde{v}_{3} \mathrm{~d} x \\
& -\mu_{2} \int_{0}^{L} z_{10}(x) \tilde{v}_{1} \mathrm{~d} x-\tilde{\mu}_{2} \int_{0}^{L} z_{20}(x) \tilde{v}_{2} \mathrm{~d} x-\tilde{\tilde{\mu}}_{2} \int_{0}^{L} z_{30}(x) \tilde{v}_{3} \mathrm{~d} x \\
& +\frac{\rho_{3}(1+\tau)}{\gamma} \int_{0}^{L}\left(\frac{\tau}{\gamma} \int_{0}^{x} f_{5} \mathrm{~d} y-f_{4}\right) \int_{0}^{x} \tilde{v}_{5} \mathrm{~d} y \mathrm{~d} x
\end{aligned}
$$

if $\tau_{i}^{\prime}(t)=0$.

If $\tau_{i}^{\prime}(t) \neq 0$, we get

$$
\begin{array}{r}
a\left(\left(v_{1}, v_{2}, v_{3}, v_{5}\right)^{T},\left(\tilde{v}_{1}, \tilde{v}_{2}, \tilde{v}_{3}, \tilde{v}_{5}\right)^{T}\right)=\tilde{a}\left(\left(\tilde{v}_{1}, \tilde{v}_{2}, \tilde{v}_{3}, \tilde{v}_{5}\right)^{T}\right), \\
\forall\left(\tilde{v}_{1}, \tilde{v}_{2}, \tilde{v}_{3}, \tilde{v}_{5}\right)^{T} \in H_{0}^{1}(0, L) \times H_{0}^{1}(0, L) \times H_{0}^{1}(0, L) \times L_{*}^{2}(0, L),
\end{array}
$$

where

$$
\begin{aligned}
& a\left(\left(v_{1}, v_{2}, v_{3}, v_{5}\right)^{T},\left(\tilde{v}_{1}, \tilde{v}_{2}, \tilde{v}_{3}, \tilde{v}_{5}\right)^{T}\right) \\
= & \int_{0}^{L}\left(k_{1}\left(\partial_{x} v_{1}+v_{2}+I v_{3}\right)\left(\partial_{x} \tilde{v}_{1}+\tilde{v}_{2}+I \tilde{v}_{3}\right) \mathrm{d} x+\int_{0}^{L} k_{3}\left(\partial_{x} v_{3}-I v_{1}\right)\left(\partial_{x} \tilde{v}_{3}-I \tilde{v}_{1}\right) \mathrm{d} x\right. \\
& +\int_{0}^{L} k_{2} \partial_{x} v_{2} \partial_{x} \tilde{v}_{2} \mathrm{~d} x+\int_{0}^{L}\left(\left(\rho_{1}+\mu_{1}+e^{\vartheta_{1 \rho}(t)}\right) v_{1} \tilde{v}_{1}+\left(\rho_{2}+\tilde{\mu}_{1}+e^{\vartheta_{2 \rho}(t)}\right) v_{2} \tilde{v}_{2}\right. \\
& \left.+\left(\rho_{1}+\tilde{\tilde{\mu}}_{1}+e^{\vartheta_{3 \rho}(t)}\right) v_{3} \tilde{v}_{3}+\frac{\tau+1}{\gamma} v_{5} \tilde{v}_{5}\right) \mathrm{d} x+\frac{\rho_{3}(1+\tau)^{2}}{\gamma^{2}} \int_{0}^{L}\left(\int_{0}^{x} v_{5} \mathrm{~d} y \int_{0}^{x} \tilde{v}_{5} \mathrm{~d} y\right) \mathrm{d} x \\
& +\int_{0}^{L}\left(-\tilde{g}_{1}^{0} \partial_{x} v_{1} \partial_{x} \tilde{v}_{1}-\tilde{g}_{2}^{0} \partial_{x} v_{2} \partial_{x} \tilde{v}_{2}-\tilde{g}_{3}^{0} \partial_{x} v_{3} \partial_{x} \tilde{v}_{3}\right) \mathrm{d} x+\frac{\sigma(1+\tau)}{\gamma} \int_{0}^{L} v_{1} \tilde{v}_{5} \mathrm{~d} x \\
& -\frac{\sigma(1+\tau)}{\gamma} \int_{0}^{L} v_{5} \tilde{v}_{1} \mathrm{~d} x
\end{aligned}
$$

the operator $\tilde{a}$ is defined by the same formula (3.23).

Now, we introduce the Hilbert space $V=H_{0}^{1}(0, L) \times H_{0}^{1}(0, L) \times H_{0}^{1}(0, L) \times L_{*}^{2}(0, L)$ equipped with the norm

$$
\left\|\left(v_{1}, v_{2}, v_{3}, v_{5}\right)\right\|_{V}^{2}=\|\left(\partial_{x} v_{1}+v_{2}+I v_{3}\left\|_{2}^{2}+\right\| \partial_{x} v_{3}-I v_{1}\left\|_{2}^{2}+\right\| \partial_{x} v_{2}\left\|_{2}^{2}+\right\| v_{5} \|_{2}^{2}\right.
$$

It is clear that $a$ and $\tilde{a}$ are bounded. Furthermore, from (2.13), we find that there exists a positive constant $c$ such that

$$
a\left(\left(v_{1}, v_{2}, v_{3}, v_{5}\right)^{T},\left(v_{1}, v_{2}, v_{3}, v_{5}\right)^{T}\right) \geq c\left\|\left(v_{1}, v_{2}, v_{3}, v_{5}\right)\right\|_{V}^{2}
$$

which implies that $a$ is coercive.

From the above, we obtain that $a$ is a bilinear continuous coercive form on $V \times V$, and $\tilde{a}$ is a linear continuous form on $V$. Therefore, using the Lax-Milgram theorem [24], we obtain that 
(3.22) and (3.25) have a unique solution $\left(v_{1}, v_{2}, v_{3}, v_{5}\right)^{T} \in V$. By substituting $v_{1}, v_{2}, v_{3}, v_{5}$ into (3.5), we obtain

$$
v_{4} \in H_{0}^{1}(0, L), v_{6} \in H_{0}^{1}(0, L), v_{7} \in H_{0}^{1}(0, L), v_{8} \in H_{0}^{1}(0, L)
$$

Next, it remains to show that

$$
v_{1}, v_{2}, v_{3} \in H^{2}(0, L) \cap H_{0}^{1}(0, L), v_{5} \in H_{*}^{1}(0, L) .
$$

Recalling (3.5) and using (3.21), we have

$$
\begin{aligned}
\left(k_{1}-\tilde{g}_{1}^{0}\right) \partial_{x x} v_{1}-I^{2} k_{3} v_{1}= & -k_{1} \partial_{x} v_{2}-I\left(k_{1}+k_{3}\right) \partial_{x} v_{3}+\sigma \partial_{x} v_{4}-\mu_{1} z_{1}(x, 1)+\left(\rho_{1}+\mu_{1}\right) v_{6}-\rho_{1} f_{6} \\
& +\left(g_{1}^{0}-\tilde{g}_{1}^{0}\right) \partial_{x x} f_{1}+\int_{0}^{+\infty} g_{1}(s) \partial_{x x}\left(\int_{0}^{s} e^{\tau-s} f_{9}(\tau) \mathrm{d} \tau\right) \mathrm{d} s \in L^{2}(0, L) .
\end{aligned}
$$

Then, by the $L^{2}$ theory for the linear elliptic equations, we obtain that

$$
v_{1} \in H^{2}(0, L) \cap H_{0}^{1}(0, L) .
$$

In the same way, we obtain

$$
v_{2} \in H^{2}(0, L) \cap H_{0}^{1}(0, L), v_{3} \in H^{2}(0, L) \cap H_{0}^{1}(0, L) .
$$

Similarly, recalling (3.6) and using (3.21), we have

$$
\partial_{x} v_{5}=-\sigma \partial_{x} v_{6}-\rho_{3} v_{4}+\rho_{3} f_{4} \in L^{2}(0, L),
$$

consequently, we get $v_{5} \in H_{*}^{1}(0, L)$.

Then, using the classical regularity theory of linear elliptic equations, we obtain a unique solution $V \in D(\mathscr{A})$ which satisfies (3.3). Hence, the operator $I d-\mathscr{A}$ is surjective.

Finally, from above, we get $\mathscr{A}$ is a maximal monotone operator. Then, by using the HilleYosida theorem [5], we obtain that if $U^{0} \in D(\mathscr{A})$, then $U \in C\left(\mathbb{R}^{+} ; D(\mathscr{A})\right) \cap C^{1}\left(\mathbb{R}^{+} ; \mathscr{H}\right)$. Moreover, it is easy to see that $D(\mathscr{A})$ is dense in $\mathscr{H}$. At last, basing on the above analysis, the well-posedness result stated in Theorem 2.1 follows from the Hille-Yosida [5, 17].

\section{Proof the Stability}

In this section, we prove Theorem 2.2. Our method builds on a suitable Lyapunov functional that can be obtained by the energy method.

Before proving our main results, we will state and prove some useful lemmas in advance.

Lemma 4.1 Let $(\varphi, \psi, w, \theta, q)$ be the solution of problem (2.9). Then the energy functional $E(t)$ defined by (2.21) satisfies

$$
\begin{aligned}
E^{\prime}(t) \leq & -c \int_{0}^{L}\left(\varphi_{t}^{2}+\psi_{t}^{2}+w_{t}^{2}\right) \mathrm{d} x-c \int_{0}^{L}\left(z_{1}^{2}(x, 1, t)+z_{2}^{2}(x, 1, t)+z_{3}^{2}(x, 1, t)\right) \mathrm{d} x \\
& +\frac{1}{2} \int_{0}^{L} \int_{0}^{+\infty}\left(g_{1}^{\prime}(s)\left(\partial_{x} \eta_{1}\right)^{2}+g_{2}^{\prime}(s)\left(\partial_{x} \eta_{2}\right)^{2}+g_{3}^{\prime}(s)\left(\partial_{x} \eta_{3}\right)^{2}\right) \mathrm{d} s \mathrm{~d} x-\int_{0}^{L} \frac{1}{\gamma} q^{2} \mathrm{~d} x \leq 0 .
\end{aligned}
$$


Proof. Multiplying the first five equations in (2.9) by $\varphi_{t}, \psi_{t}, w_{t}, \theta$ and $q$, respectively, integrating over $(0, L)$, using integration by parts and the boundary conditions, we get

$$
\begin{aligned}
& \quad \frac{1}{2} \frac{d}{d t}\left[\int _ { 0 } ^ { L } \left(\rho_{1} \varphi_{t}^{2}+\rho_{2} \psi_{t}^{2}+\rho_{1} w_{t}^{2}+k_{2} \psi_{x}^{2}+k_{1}\left(\varphi_{x}+\psi+I w\right)^{2}+k_{3}\left(w_{x}-I \varphi\right)^{2}+\rho_{3} \theta^{2}\right.\right. \\
& \left.\left.+\frac{\tau}{\gamma} q^{2}\right) \mathrm{~d} x-\int_{0}^{L}\left(g_{1}^{0} \varphi_{x}^{2}+g_{2}^{0} \psi_{x}^{2}+g_{3}^{0} w_{x}^{2}\right) \mathrm{d} x+\left\|\eta_{1}\right\|_{H_{1}^{*}}^{2}+\left\|\eta_{2}\right\|_{H_{2}^{*}}^{2}+\left\|\eta_{3}\right\|_{H_{3}^{*}}^{2}\right] \\
& =\frac{1}{2} \int_{0}^{L} \int_{0}^{+\infty}\left(g_{1}^{\prime}(s)\left(\partial_{x} \eta_{1}\right)^{2}+g_{2}^{\prime}(s)\left(\partial_{x} \eta_{2}\right)^{2}+g_{3}^{\prime}(s)\left(\partial_{x} \eta_{3}\right)^{2}\right) \mathrm{d} s \mathrm{~d} x-\frac{1}{\gamma} \int_{0}^{L} q^{2} \mathrm{~d} x .
\end{aligned}
$$

Then, multiplying the last three equations in (2.9) by $\zeta_{1} z_{1}, \zeta_{2} z_{2}$ and $\zeta_{3} z_{3}$ respectively, integrating the resulting equations over $(0, L) \times(0,1)$ with respect to $x$ and $\rho$, we obtain

$$
\begin{aligned}
& \frac{1}{2} \frac{d}{d t} \int_{0}^{L} \int_{0}^{1}\left(\zeta_{1} \tau_{1}(t) z_{1}^{2}(x, \rho, t)+\zeta_{2} \tau_{2}(t) z_{2}^{2}(x, \rho, t)+\zeta_{3} \tau_{3}(t) z_{3}^{2}(x, \rho, t)\right) \mathrm{d} \rho \mathrm{d} x \\
= & \frac{1}{2} \int_{0}^{L} \int_{0}^{1}\left(\zeta_{1} \tau_{1}^{\prime}(t) z_{1}^{2}(x, \rho, t)+\zeta_{2} \tau_{2}^{\prime}(t) z_{2}^{2}(x, \rho, t)+\zeta_{3} \tau_{3}^{\prime}(t) z_{3}^{2}(x, \rho, t)\right) \mathrm{d} \rho \mathrm{d} x \\
& -\int_{0}^{L} \int_{0}^{1}\left(\zeta_{1}\left(1-\tau_{1}^{\prime}(t) \rho\right) z_{1}(x, \rho, t) z_{1}(x, \rho, t)+\zeta_{2}\left(1-\tau_{2}^{\prime}(t) \rho\right) z_{2}(x, \rho, t) z_{2}(x, \rho, t)\right. \\
& \left.+\zeta_{3}\left(1-\tau_{3}^{\prime}(t) \rho\right) z_{3}(x, \rho, t) z_{3 \rho}(x, \rho, t)\right) \mathrm{d} \rho \mathrm{d} x . \\
= & -\frac{1}{2} \int_{0}^{L} \int_{0}^{1} \frac{\partial}{\partial_{\rho}}\left(\left(1-\tau_{1}^{\prime}(t) \rho\right) z_{1}^{2}(x, \rho, t)+\left(1-\tau_{2}^{\prime}(t) \rho\right) z_{2}^{2}(x, \rho, t)+\left(1-\tau_{3}^{\prime}(t) \rho\right) z_{3}^{2}(x, \rho, t)\right) \mathrm{d} \rho \mathrm{d} x \\
= & \frac{1}{2} \int_{0}^{L}\left(\zeta_{1} \varphi_{t}^{2}(x, t)+\zeta_{2} \psi_{t}^{2}(x, t)+\zeta_{3} w_{t}^{2}(x, t)\right) \mathrm{d} x \\
& -\frac{1}{2} \int_{0}^{L}\left(\zeta_{1}\left(1-\tau_{1}^{\prime}(t)\right) z_{1}^{2}(x, 1, t)+\zeta_{2}\left(1-\tau_{2}^{\prime}(t)\right) z_{2}^{2}(x, 1, t)+\zeta_{3}\left(1-\tau_{3}^{\prime}(t)\right) z_{3}^{2}(x, 1, t)\right) \mathrm{d} x .
\end{aligned}
$$

From (4.2) and (4.3), we have

$$
\begin{aligned}
\frac{d E(t)}{d t}= & -\left(\mu_{1}-\frac{\zeta_{1}}{2}\right) \int_{0}^{L} \varphi_{t}^{2} \mathrm{~d} x-\left(\tilde{\mu}_{1}-\frac{\zeta_{2}}{2}\right) \int_{0}^{L} \psi_{t}^{2} \mathrm{~d} x-\left(\tilde{\tilde{\mu}}_{1}-\frac{\zeta_{3}}{2}\right) \int_{0}^{L} w_{t}^{2} \mathrm{~d} x \\
& -\mu_{2} \int_{0}^{L} z_{1}(x, 1, t) \varphi_{t} \mathrm{~d} x-\tilde{\mu}_{2} \int_{0}^{L} z_{2}(x, 1, t) \psi_{t} \mathrm{~d} x-\tilde{\tilde{\mu}}_{2} \int_{0}^{L} z_{3}(x, 1, t) w_{t} \mathrm{~d} x \\
& -\frac{1}{2} \int_{0}^{L} \int_{0}^{+\infty} g_{1}(s) \partial_{s}\left(\partial_{x} \eta_{1}\right)^{2} \mathrm{~d} s \mathrm{~d} x-\frac{1}{2} \int_{0}^{L} \int_{0}^{+\infty} g_{2}(s) \partial_{s}\left(\partial_{x} \eta_{2}\right)^{2} \mathrm{~d} s \mathrm{~d} x \\
& -\frac{1}{2} \int_{0}^{L} \int_{0}^{+\infty} g_{3}(s) \partial_{s}\left(\partial_{x} \eta_{3}\right)^{2} \mathrm{~d} s \mathrm{~d} x-\int_{0}^{L} \frac{1}{\gamma} q^{2} \mathrm{~d} x \\
& -\sum_{i=1}^{3} \frac{\zeta_{i}\left(1-\tau_{i}^{\prime}(t)\right)}{2} \int_{0}^{L} z_{i}^{2}(x, 1, t) \mathrm{d} x .
\end{aligned}
$$

Finally, by using (2.3), (2.17) and Young's inequality, we obtain (4.1) (note that $g_{i}$ is nonincreasing).

As in [14], let us define the functionals:

$$
I_{1}(t)=-\rho_{1} \int_{0}^{L} \varphi_{t} \int_{0}^{+\infty} g_{1}(s) \eta_{1} \mathrm{~d} s \mathrm{~d} x
$$




$$
I_{2}(t)=-\rho_{2} \int_{0}^{L} \psi_{t} \int_{0}^{+\infty} g_{2}(s) \eta_{2} \mathrm{~d} s \mathrm{~d} x
$$

and

$$
I_{3}(t)=-\rho_{3} \int_{0}^{L} w_{t} \int_{0}^{+\infty} g_{3}(s) \eta_{3} \mathrm{~d} s \mathrm{~d} x .
$$

Lemma 4.2 The functionals $I_{i}(i=1,2,3)$ satisfy, for any $\delta>0$,

$$
\begin{aligned}
I_{1}^{\prime}(t) \leq & -\rho_{1}\left(g_{1}^{0}-\left(1+\frac{1}{\rho_{1}}\right) \delta\right) \int_{0}^{L} \varphi_{t}^{2} \mathrm{~d} x \\
& +\delta \int_{0}^{L}\left(\psi_{x}^{2}+\left(\varphi_{x}+\psi+I w\right)^{2}+\left(w_{x}-I \varphi\right)^{2}+\theta^{2}+z_{1}^{2}(x, 1, t)\right) \mathrm{d} x \\
& +c_{\delta} \int_{0}^{L} \int_{0}^{+\infty} g_{1}(s)\left(\partial_{x} \eta_{1}\right)^{2} \mathrm{~d} s \mathrm{~d} x-c_{\delta} \int_{0}^{L} \int_{0}^{+\infty} g_{1}^{\prime}(s)\left(\partial_{x} \eta_{1}\right)^{2} \mathrm{~d} s \mathrm{~d} x \\
I_{2}^{\prime}(t) \leq & -\rho_{2}\left(g_{2}^{0}-\left(1+\frac{1}{\rho_{2}}\right) \delta\right) \int_{0}^{L} \psi_{t}^{2} \mathrm{~d} x \\
& +\delta \int_{0}^{L}\left(\psi_{x}^{2}+\left(\varphi_{x}+\psi+I w\right)^{2}+\left(w_{x}-I \varphi\right)^{2}+z_{2}^{2}(x, 1, t)\right) \mathrm{d} x \\
& +c_{\delta} \int_{0}^{L} \int_{0}^{+\infty} g_{2}(s)\left(\partial_{x} \eta_{2}\right)^{2} \mathrm{~d} s \mathrm{~d} x-c_{\delta} \int_{0}^{L} \int_{0}^{+\infty} g_{2}^{\prime}(s)\left(\partial_{x} \eta_{2}\right)^{2} \mathrm{~d} s \mathrm{~d} x
\end{aligned}
$$

and

$$
\begin{aligned}
I_{3}^{\prime}(t) \leq & -\rho_{1}\left(g_{3}^{0}-\left(1+\frac{1}{\rho_{1}}\right) \delta\right) \int_{0}^{L} w_{t}^{2} \mathrm{~d} x \\
& +\delta \int_{0}^{L}\left(\psi_{x}^{2}+\left(\varphi_{x}+\psi+I w\right)^{2}+\left(w_{x}-I \varphi\right)^{2}+z_{2}^{3}(x, 1, t)\right) \mathrm{d} x \\
& +c_{\delta} \int_{0}^{L} \int_{0}^{+\infty} g_{3}(s)\left(\partial_{x} \eta_{3}\right)^{2} \mathrm{~d} s \mathrm{~d} x-c_{\delta} \int_{0}^{L} \int_{0}^{+\infty} g_{3}^{\prime}(s)\left(\partial_{x} \eta_{3}\right)^{2} \mathrm{~d} s \mathrm{~d} x
\end{aligned}
$$

where $g_{i}^{0}$ is defined by (2.15) and $c_{\delta}$ is a positive constant depending on $\delta$.

Proof. Differentiating $I_{1}$ with respect to $t$, using the first equation of problem (2.9), integrating by parts and using the fact that

$$
\begin{aligned}
\partial_{t} \int_{0}^{+\infty} g_{1}(s) \eta_{1} \mathrm{~d} s & =\partial_{t} \int_{0}^{+\infty} g_{1}(t-s)(\varphi(t)-\varphi(s)) \mathrm{d} s \\
& =\int_{0}^{+\infty} g_{1}^{\prime}(t-s)(\varphi(t)-\varphi(s)) \mathrm{d} s+\left(\int_{0}^{+\infty} g_{1}(t-s) \mathrm{d} s\right) \varphi_{t} \\
& =\int_{0}^{+\infty} g_{1}^{\prime}(s) \eta_{1} \mathrm{~d} s+g_{1}^{0} \varphi_{t}
\end{aligned}
$$

we obtain

$$
\begin{aligned}
I_{1}^{\prime}(t)= & -\rho_{1} g_{1}^{0} \int_{0}^{L} \varphi_{t}^{2} \mathrm{~d} x-\rho_{1} \int_{0}^{L} \varphi_{t} \int_{0}^{+\infty} g_{1}^{\prime}(s) \eta_{1} \mathrm{~d} s \mathrm{~d} x-\sigma \int_{0}^{L} \theta \int_{0}^{+\infty} g_{1}(s) \partial_{x} \eta_{1} \mathrm{~d} s \mathrm{~d} x \\
& +k_{1} \int_{0}^{L}\left(\varphi_{x}+\psi+I w\right) \int_{0}^{+\infty} g_{1}(s) \partial_{x} \eta_{1} \mathrm{~d} s \mathrm{~d} x-I k_{3} \int_{0}^{L}\left(w_{x}-I \varphi\right) \int_{0}^{+\infty} g_{1}(s) \eta_{1} \mathrm{~d} s \mathrm{~d} x
\end{aligned}
$$




$$
\begin{aligned}
& -g_{1}^{0} \int_{0}^{L} \varphi_{x}\left(\int_{0}^{+\infty} g_{1}(s) \partial_{x} \eta_{1} \mathrm{~d} s\right) \mathrm{d} x+\int_{0}^{L}\left(\int_{0}^{+\infty} g_{1}(s) \partial_{x} \eta_{1} \mathrm{~d} s\right)^{2} \mathrm{~d} x \\
& +\mu_{1} \int_{0}^{L} \varphi_{t} \int_{0}^{+\infty} g_{1}(s) \eta_{1} \mathrm{~d} s \mathrm{~d} x+\mu_{2} \int_{0}^{L} z_{1}(x, 1, t) \int_{0}^{+\infty} g_{1}(s) \eta_{1} \mathrm{~d} s \mathrm{~d} x .
\end{aligned}
$$

Using Young's, Poincaré's and Hölder's inequalities for the last eight terms of this equality, we get

$$
\begin{aligned}
I_{1}^{\prime}(t) \leq & -\rho_{1} g_{1}^{0} \int_{0}^{L} \varphi_{t}^{2} \mathrm{~d} x+\varepsilon \rho_{1} \int_{0}^{L} \varphi_{t}^{2} \mathrm{~d} x \\
& -\frac{\rho_{1} c}{4 \varepsilon} \int_{0}^{L} \int_{0}^{+\infty} g_{1}^{\prime}(s)\left(\partial_{x} \eta_{1}\right)^{2} \mathrm{~d} s \mathrm{~d} x+\varepsilon k_{1} \int_{0}^{L}\left(\varphi_{x}+\psi+I w\right)^{2} \mathrm{~d} x \\
& +\frac{k_{1} g_{1}^{0}}{4 \varepsilon} \int_{0}^{L} \int_{0}^{+\infty} g_{1}(s)\left(\partial_{x} \eta_{1}\right)^{2} \mathrm{~d} s \mathrm{~d} x+\varepsilon I k_{3} \int_{0}^{L}\left(w_{x}-I \varphi\right)^{2} \mathrm{~d} x \\
& +\frac{I k_{3} c g_{1}^{0}}{4 \varepsilon} \int_{0}^{L} \int_{0}^{+\infty} g_{1}(s)\left(\partial_{x} \eta_{1}\right)^{2} \mathrm{~d} s \mathrm{~d} x+\varepsilon g_{1}^{0} \int_{0}^{L} \varphi_{x}^{2} \mathrm{~d} x \\
& +\frac{\left(g_{1}^{0}\right)^{2}}{4 \varepsilon} \int_{0}^{L} \int_{0}^{+\infty} g_{1}(s)\left(\partial_{x} \eta_{1}\right)^{2} \mathrm{~d} s \mathrm{~d} x+\varepsilon \sigma \int_{0}^{L} \theta^{2} \mathrm{~d} x \\
& +\frac{\sigma g_{1}^{0}}{4 \varepsilon} \int_{0}^{L} \int_{0}^{+\infty} g_{1}(s)\left(\partial_{x} \eta_{1}\right)^{2} \mathrm{~d} s \mathrm{~d} x+g_{1}^{0} \int_{0}^{L} \int_{0}^{+\infty} g_{1}(s)\left(\partial_{x} \eta_{1}\right)^{2} \mathrm{~d} s \mathrm{~d} x \\
& +\varepsilon \mu_{1} \int_{0}^{L} \varphi_{t}^{2} \mathrm{~d} x+\frac{\mu_{1} c g_{1}^{0}}{4 \varepsilon} \int_{0}^{L} \int_{0}^{+\infty} g_{1}(s)\left(\partial_{x} \eta_{1}\right)^{2} \mathrm{~d} s \mathrm{~d} x \\
& +\varepsilon\left|\mu_{2}\right| \int_{0}^{L} z_{1}^{2}(x, 1, t) \mathrm{d} x+\frac{\left|\mu_{2}\right| c g_{1}^{0}}{4 \varepsilon} \int_{0}^{L} \int_{0}^{+\infty} g_{1}(s)\left(\partial_{x} \eta_{1}\right)^{2} \mathrm{~d} s \mathrm{~d} x .
\end{aligned}
$$

By using (2.14) to estimate $\int_{0}^{L} \varphi_{x}^{2} \mathrm{~d} x$ and choosing

$$
\begin{gathered}
\delta=\max \left\{\varepsilon\left(\frac{k_{1} g_{1}^{0}}{\bar{k}_{0}}+k_{1}\right), \frac{\varepsilon g_{1}^{0} k_{2}}{\bar{k}_{0}}, \varepsilon\left(\frac{g_{1}^{0} k_{3}}{\bar{k}_{0}}+I k_{3}\right), \varepsilon \sigma, \varepsilon, \varepsilon \mu_{1}, \varepsilon\left|\mu_{2}\right|\right\}, \\
c_{\delta}=\max \left\{\frac{\rho_{1} c}{4 \varepsilon}, \frac{k_{1} g_{1}^{0}}{4 \varepsilon}+\frac{I k_{3} g_{1}^{0} c}{4 \varepsilon}+\frac{\left(g_{1}^{0}\right)^{2}}{4 \varepsilon}+\frac{\sigma g_{1}^{0}}{4 \varepsilon}+g_{1}^{0}+\frac{\mu_{1} c g_{1}^{0}}{4 \varepsilon}+\frac{\left|\mu_{2}\right| c g_{1}^{0}}{4 \varepsilon}\right\},
\end{gathered}
$$

(4.4) is established. Similarly, using the second and the third equations of problem (2.9), we obtain (4.5) and (4.6).

Lemma 4.3 There exist positive constants $c_{1}$ and $c_{2}$ such that the functional

$$
I_{4}(t)=\int_{0}^{L}\left(\rho_{1} \varphi \varphi_{t}+\rho_{2} \psi \psi_{t}+\rho_{1} w w_{t}\right) \mathrm{d} x
$$

satisfies

$$
\begin{aligned}
I_{4}^{\prime}(t) \leq & \int_{0}^{L}\left(\left(\rho_{1}+c_{2}\right) \varphi_{t}^{2}+\left(\rho_{2}+c_{2}\right) \psi_{t}^{2}+\left(\rho_{1}+c_{2}\right) w_{t}^{2}\right) \mathrm{d} x \\
& -c_{1} \int_{0}^{L}\left(\psi_{x}^{2}+\left(\varphi_{x}+\psi+I w\right)^{2}+\left(w_{x}-I \varphi\right)^{2}\right) \mathrm{d} x+c_{2} \int_{0}^{L} \theta^{2} \mathrm{~d} x \\
& +c_{2} \int_{0}^{L} \int_{0}^{+\infty}\left(g_{1}(s)\left(\partial_{x} \eta_{1}\right)^{2}+g_{2}(s)\left(\partial_{x} \eta_{2}\right)^{2}+g_{3}(s)\left(\partial_{x} \eta_{3}\right)^{2}\right) \mathrm{d} s \mathrm{~d} x \\
& +c_{2} \int_{0}^{L}\left(z_{1}^{2}(x, 1, t)+z_{2}^{2}(x, 1, t)+z_{3}^{2}(x, 1, t)\right) \mathrm{d} x .
\end{aligned}
$$


Proof. By exploiting first three equations of problem (2.9) and integrating by parts, we have

$$
\begin{aligned}
I_{4}^{\prime}(t)= & \int_{0}^{L}\left(\rho_{1} \varphi_{t}^{2}+\rho_{2} \psi_{t}^{2}+\rho_{1} w_{t}^{2}\right) \mathrm{d} x-k_{1} \int_{0}^{L}\left(\varphi_{x}+\psi+I w\right)^{2} \mathrm{~d} x \\
& -k_{3} \int_{0}^{L}\left(w_{x}-I \varphi\right)^{2} \mathrm{~d} x+g_{1}^{0} \int_{0}^{L} \varphi_{x}^{2} \mathrm{~d} x-\left(k_{2}-g_{2}^{0}\right) \int_{0}^{L} \psi_{x}^{2} \mathrm{~d} x+g_{3}^{0} \int_{0}^{L} w_{x}^{2} \mathrm{~d} x \\
& -\int_{0}^{L} \varphi_{x} \int_{0}^{+\infty} g_{1}(s) \partial_{x} \eta_{1} \mathrm{~d} s \mathrm{~d} x-\int_{0}^{L} \psi_{x} \int_{0}^{+\infty} g_{2}(s) \partial_{x} \eta_{2} \mathrm{~d} s \mathrm{~d} x \\
& -\int_{0}^{L} w_{x} \int_{0}^{+\infty} g_{3}(s) \partial_{x} \eta_{3} \mathrm{~d} s \mathrm{~d} x+\sigma \int_{0}^{L} \varphi_{x} \theta \mathrm{d} x \\
& -\mu_{1} \int_{0}^{L} \varphi \varphi_{t} \mathrm{~d} x-\tilde{\mu}_{1} \int_{0}^{L} \psi \psi_{t} \mathrm{~d} x-\tilde{\tilde{\mu}}_{1} \int_{0}^{L} w w_{t} \mathrm{~d} x \\
& -\mu_{2} \int_{0}^{L} z_{1}(x, 1, t) \varphi \mathrm{d} x-\tilde{\mu}_{2} \int_{0}^{L} z_{2}(x, 1, t) \psi \mathrm{d} x-\tilde{\tilde{\mu}}_{2} \int_{0}^{L} z_{3}(x, 1, t) w \mathrm{~d} x .
\end{aligned}
$$

Using Young's Poincaré's and Hölder's inequalities for the last ten terms in the right hand side of (4.8), we get, for any $\epsilon>0$, there exists a positive constant $c_{\epsilon}$ such that

$$
\begin{aligned}
& \quad-\int_{0}^{L} \varphi_{x} \int_{0}^{+\infty} g_{1}(s) \partial_{x} \eta_{1} \mathrm{~d} s \mathrm{~d} x-\int_{0}^{L} \psi_{x} \int_{0}^{+\infty} g_{2}(s) \partial_{x} \eta_{2} \mathrm{~d} s \mathrm{~d} x-\int_{0}^{L} w_{x} \int_{0}^{+\infty} g_{3}(s) \partial_{x} \eta_{3} \mathrm{~d} s \mathrm{~d} x \\
& \quad+\sigma \int_{0}^{L} \varphi_{x} \theta \mathrm{d} x-\mu_{1} \int_{0}^{L} \varphi \varphi_{t} \mathrm{~d} x-\tilde{\mu}_{1} \int_{0}^{L} \psi \psi_{t} \mathrm{~d} x-\tilde{\tilde{\mu}}_{1} \int_{0}^{L} w w_{t} \mathrm{~d} x \\
& \quad-\mu_{2} \int_{0}^{L} z_{1}(x, 1, t) \varphi \mathrm{d} x-\tilde{\mu}_{2} \int_{0}^{L} z_{2}(x, 1, t) \psi \mathrm{d} x-\tilde{\tilde{\mu}}_{2} \int_{0}^{L} z_{3}(x, 1, t) w \mathrm{~d} x \\
& \leq \epsilon \int_{0}^{L}\left(\varphi_{x}^{2}+\psi_{x}^{2}+w_{x}^{2}\right) \mathrm{d} x+c_{\epsilon} \int_{0}^{L} \int_{0}^{+\infty}\left(g_{1}(s)\left(\partial_{x} \eta_{1}\right)^{2}+g_{2}(s)\left(\partial_{x} \eta_{2}\right)^{2}+g_{3}(s)\left(\partial_{x} \eta_{3}\right)^{2}\right) \mathrm{d} s \mathrm{~d} x \\
& \quad+c_{\epsilon} \int_{0}^{L} \theta^{2} \mathrm{~d} x+c_{\epsilon} \int_{0}^{L}\left(\varphi_{t}^{2}+\psi_{t}^{2}+w_{t}^{2}\right) \mathrm{d} x+c_{\epsilon} \int_{0}^{L}\left(z_{1}^{2}(x, 1, t)+z_{2}^{2}(x, 1, t)+z_{3}^{2}(x, 1, t)\right) \mathrm{d} x .
\end{aligned}
$$

Inserting this inequality into (4.8) and using (2.13), we get

$$
\begin{aligned}
I_{4}^{\prime}(t) \leq & \int_{0}^{L}\left(\left(p_{1}+c_{\epsilon}\right) \varphi_{t}^{2}+\left(p_{2}+c_{\epsilon}\right) \psi_{t}^{2}+\left(p_{1}+c_{\epsilon}\right) w_{t}^{2}\right) \mathrm{d} x-\left(k_{0}-\epsilon\right) \int_{0}^{L}\left(\varphi_{x}^{2}+\psi_{x}^{2}+w_{x}^{2}\right) \mathrm{d} x \\
& +c_{\epsilon} \int_{0}^{L} \int_{0}^{+\infty}\left(g_{1}(s)\left(\partial_{x} \eta_{1}\right)^{2}+g_{2}(s)\left(\partial_{x} \eta_{2}\right)^{2}+g_{3}(s)\left(\partial_{x} \eta_{3}\right)^{2}\right) \mathrm{d} s \mathrm{~d} x+c_{\epsilon} \int_{0}^{L} \theta^{2} \mathrm{~d} x \\
& +c_{\epsilon} \int_{0}^{L}\left(z_{1}^{2}(x, 1, t)+z_{2}^{2}(x, 1, t)+z_{3}^{2}(x, 1, t)\right) \mathrm{d} x
\end{aligned}
$$

Then, choosing $0<\epsilon<k_{0}$ and inserting (2.16) in (4.9), we get (4.7) with $c_{1}=\frac{\left(k_{0}-\epsilon\right)}{\bar{k}_{0}}$ and $c_{2}=c_{\epsilon}$.

Lemma 4.4 ([4]) For any $\varepsilon_{3}>0$, the functional

$$
I_{5}(t)=\tau \rho_{3} \int_{0}^{L} \theta \int_{0}^{x} q(y) \mathrm{d} y \mathrm{~d} x
$$

satisfies

$$
I_{5}^{\prime}(t) \leq-\frac{\gamma \rho_{3}}{2} \int_{0}^{L} \theta^{2} \mathrm{~d} x+\varepsilon_{3} \int_{0}^{L} \varphi_{t}^{2} \mathrm{~d} x+c\left(1+\frac{1}{\varepsilon_{3}}\right) \int_{0}^{L} q^{2} \mathrm{~d} x
$$


Proof. Taking the derivative of $I_{5}$ with respect to $t$, using the fourth and fifth equations of problem (2.9) and integrating by parts, we get

$$
I_{5}^{\prime}(t)=-\gamma p_{3} \int_{0}^{L} \theta^{2} \mathrm{~d} x+\tau \int_{0}^{L} q^{2} \mathrm{~d} x+\tau \sigma \int_{0}^{L} q \varphi_{t} \mathrm{~d} x-p_{3} \int_{0}^{L} \theta \int_{0}^{x} q(y) \mathrm{d} y \mathrm{~d} x .
$$

Using Cauchy-Schwarz's and Young's inequalities with $\varepsilon_{3}>0$, we get (4.10).

Lemma 4.5 The functionals

$$
\begin{aligned}
& I_{6}=\zeta_{1} \tau_{1}(t) \int_{0}^{L} \int_{0}^{1} e^{-2 \tau_{1}(t) \rho} z_{1}^{2}(x, \rho, t) \mathrm{d} \rho \mathrm{d} x, \\
& I_{7}=\zeta_{2} \tau_{2}(t) \int_{0}^{L} \int_{0}^{1} e^{-2 \tau_{1}(t) \rho} z_{2}^{2}(x, \rho, t) \mathrm{d} \rho \mathrm{d} x, \\
& I_{8}=\zeta_{3} \tau_{3}(t) \int_{0}^{L} \int_{0}^{1} e^{-2 \tau_{1}(t) \rho} z_{3}^{2}(x, \rho, t) \mathrm{d} \rho \mathrm{d} x
\end{aligned}
$$

satisfies

$$
\begin{aligned}
& I_{6}^{\prime}(t) \leq-2 I_{6}(t)+\zeta_{1} \int_{0}^{l} \varphi_{t}^{2} \mathrm{~d} x, \\
& I_{7}^{\prime}(t) \leq-2 I_{7}(t)+\zeta_{2} \int_{0}^{l} \psi_{t}^{2} \mathrm{~d} x, \\
& I_{8}^{\prime}(t) \leq-2 I_{8}(t)+\zeta_{3} \int_{0}^{l} w_{t}^{2} \mathrm{~d} x .
\end{aligned}
$$

Proof. Taking the derivative of $I_{6}$ with respect to $t$, we get

$$
\begin{aligned}
I_{6}^{\prime}(t)= & \zeta_{1} \tau_{1}^{\prime}(t) \int_{0}^{L} \int_{0}^{1} e^{-2 \tau_{1}(t) \rho} z_{1}^{2}(x, \rho, t) \mathrm{d} \rho \mathrm{d} x-2 \zeta_{1} \tau_{1}(t) \tau_{1}^{\prime}(t) \int_{0}^{L} \int_{0}^{1} e^{-2 \tau_{1}(t) \rho} \rho z_{1}^{2}(x, \rho, t) \mathrm{d} \rho \mathrm{d} x \\
& +2 \zeta_{1} \tau_{1}(t) \int_{0}^{L} \int_{0}^{1} e^{-2 \tau_{1}(t) \rho} z_{1} z_{1 t}(x, \rho, t) \mathrm{d} \rho \mathrm{d} x .
\end{aligned}
$$

By using (2.8), the last term in (4.14) can be rewritten as follows

$$
\left.2 \zeta_{1} \tau_{1}(t) \int_{0}^{L} \int_{0}^{1} e^{-2 \tau_{1}(t) \rho} z_{1} z_{1 t}(x, \rho, t) \mathrm{d} \rho \mathrm{d} x=-2 \zeta_{1} \int_{0}^{L} \int_{0}^{1} e^{-2 \tau_{1}(t) \rho}\left(1-\tau_{1}^{\prime}(t) \rho\right) z_{1} z_{1 \rho}(x, \rho, t)\right) \mathrm{d} \rho \mathrm{d} x .
$$

Also, one can see that

$$
\begin{aligned}
& -2 \zeta_{1} \int_{0}^{L} \int_{0}^{1} e^{-2 \tau_{1}(t) \rho}\left(1-\tau_{1}^{\prime}(t) \rho\right) z_{1} z_{1 \rho}(x, \rho, t) \mathrm{d} \rho \mathrm{d} x \\
= & -\zeta_{1} \int_{0}^{L} \int_{0}^{1} \frac{\partial}{\partial \rho}\left(e^{-2 \tau_{1}(t) \rho}\left(1-\tau_{1}^{\prime}(t) \rho\right) z_{1}^{2}(x, \rho, t)\right) \mathrm{d} \rho \mathrm{d} x \\
& -2 \zeta_{1} \tau_{1}(t) \int_{0}^{L} \int_{0}^{1} e^{-2 \tau_{1}(t) \rho}\left(1-\tau_{1}^{\prime}(t) \rho\right) z_{1}^{2}(x, \rho, t) \mathrm{d} \rho \mathrm{d} x
\end{aligned}
$$




$$
-\zeta_{1} \tau_{1}^{\prime}(t) \int_{0}^{L} \int_{0}^{1} e^{-2 \tau_{1}(t) \rho} z_{1}^{2}(x, \rho, t) \mathrm{d} \rho \mathrm{d} x .
$$

Using (4.15) and (4.16), equation (4.14) takes the form

$$
\begin{aligned}
I_{6}^{\prime}(t)= & -2 \zeta_{1} \tau_{1}(t) \int_{0}^{L} \int_{0}^{1} e^{-2 \tau_{1}(t) \rho} z_{1}^{2}(x, \rho, t) \mathrm{d} \rho \mathrm{d} x+\zeta_{1} \int_{0}^{L} \varphi_{t}^{2} \mathrm{~d} x \\
& -\zeta_{1}\left(1-\tau_{1}^{\prime}(t)\right) e^{-2 \tau_{1}(t)} \int_{0}^{1} z_{1}^{2}(x, \rho, t) \mathrm{d} \rho \mathrm{d} x,
\end{aligned}
$$

from which immediately follows (4.11). Similarly, we get (4.12) and (4.13) in the same way.

Now, let $N_{1}, N_{2}, N_{3}>0$, and

$$
L(t)=N_{1} E+N_{2}\left(I_{1}+I_{2}+I_{3}\right)+I_{4}+N_{3} I_{5}+I_{6}+I_{7}+I_{8}
$$

First, taking the derivative of $L(t)$ with respect to $t$, using (4.4)-(4.6) with $\delta=\frac{1}{N_{2}^{2}},(4.7),(4.10)$, (4.11), (4.12) and (4.13), we get

$$
\begin{aligned}
L^{\prime}(t) \leq & -\left(c_{1}-\frac{3}{N_{2}}\right) \int_{0}^{L}\left(\psi_{x}^{2}+\left(\varphi_{x}+\psi+I w\right)^{2}+\left(w_{x}-I \varphi\right)^{2}\right) \mathrm{d} x \\
& -\left(N_{1} c+\rho_{1}\left(N_{2} g_{1}^{0}-\left(1+\frac{1}{\rho_{1}}\right) \frac{1}{N_{2}}-1\right)-c_{2}-N_{3} \varepsilon_{3}-\zeta_{1}\right) \int_{0}^{L} \varphi_{t}^{2} \mathrm{~d} x \\
& -\left(N_{2} c+\rho_{2}\left(N_{2} g_{2}^{0}-\left(1+\frac{1}{\rho_{2}}\right) \frac{1}{N_{2}}-1\right)-c_{2}-\zeta_{2}\right) \int_{0}^{L} \psi_{t}^{2} \mathrm{~d} x \\
& -\left(N_{3} c+\rho_{1}\left(N_{2} g_{3}^{0}-\left(1+\frac{1}{\rho_{1}}\right) \frac{1}{N_{2}}-1\right)-c_{2}-\zeta_{3}\right) \int_{0}^{L} w_{t}^{2} \mathrm{~d} x \\
& -\left(\frac{N_{3} \gamma \rho_{3}}{2}-\frac{1}{N_{2}}-c_{2}\right) \int_{0}^{L} \theta^{2} \mathrm{~d} x-\left(\frac{N_{1}}{\gamma}-N_{3} c\left(1+\frac{1}{\varepsilon_{3}}\right)\right) \int_{0}^{L} q^{2} \mathrm{~d} x \\
& -\left(N_{1} c-\frac{1}{N_{2}}-c_{2}\right) \int_{0}^{L} z_{1}^{2}(x, 1, t) \mathrm{d} x-\left(N_{1} c-\frac{1}{N_{2}}-c_{2}\right) \int_{0}^{L} z_{2}^{2}(x, 1, t) \mathrm{d} x \\
& -\left(N_{1} c-\frac{1}{N_{2}}-c_{2}\right) \int_{0}^{L} z_{3}^{2}(x, 1, t) \mathrm{d} x-2 I_{6}(t)-2 I_{7}(t)-2 I_{8}(t) \\
& +\left(\frac{N_{1}}{2}-c_{N_{2}}\right) \int_{0}^{L} \int_{0}^{+\infty}\left(g_{1}^{\prime}(s)\left(\partial_{x} \eta_{1}\right)^{2}+g_{2}^{\prime}(s)\left(\partial_{x} \eta_{2}\right)^{2}+g_{3}^{\prime}(s)\left(\partial_{x} \eta_{3}\right)^{2}\right) \mathrm{d} s \mathrm{~d} x \\
& +c_{N_{2}} \int_{0}^{L} \int_{0}^{+\infty}\left(g_{1}(s)\left(\partial_{x} \eta_{1}\right)^{2}+g_{2}(s)\left(\partial_{x} \eta_{2}\right)^{2}+g_{3}(s)\left(\partial_{x} \eta_{3}\right)^{2}\right) \mathrm{d} s \mathrm{~d} x,
\end{aligned}
$$

where $c_{N_{2}}=N_{2} c_{\delta}+c_{2}$. At this point, we choose $N_{3}$ large enough so that

$$
\frac{N_{3} \gamma \rho_{3}}{2}-c_{2}>0
$$

Then, we choose $\varepsilon$ small enough and $N_{2}$ large enough (note that $g_{i}$ is continuous non-negative and $\left.g_{i}(0)>0\right)$ so that

$$
\begin{gathered}
c_{1}-\frac{3}{N_{2}}>0, \frac{N_{3} \gamma \rho_{3}}{2}-\frac{1}{N_{2}}-c_{2}>0, \rho_{1}\left(N_{2} g_{1}^{0}-\left(1+\frac{1}{\rho_{1}}\right) \frac{1}{N_{2}}-1\right)-c_{2}-N_{3} \varepsilon_{3}-\zeta_{1}>0, \\
\rho_{2}\left(N_{2} g_{2}^{0}-\left(1+\frac{1}{\rho_{2}}\right) \frac{1}{N_{2}}-1\right)-c_{2}-\zeta_{2}>0, \rho_{1}\left(N_{2} g_{3}^{0}-\left(1+\frac{1}{\rho_{1}}\right) \frac{1}{N_{2}}-1\right)-c_{2}-\zeta_{3}>0 .
\end{gathered}
$$


Finally, we choose $N_{1}$ large enough so that

$$
N_{1} c-\frac{1}{N_{2}}-c_{2}>0, \frac{N_{1}}{\gamma}-N_{3} c\left(1+\frac{1}{\varepsilon_{3}}\right)>0 .
$$

From the above, we deduce that there exist positive constants $c_{3}$ and $c_{4}$ such that (4.18) becomes

$$
\begin{aligned}
L^{\prime}(t) \leq & -c_{3} E(t)+\left(\frac{N_{1}}{2}-c_{4}\right) \int_{0}^{L} \int_{0}^{+\infty}\left(g_{1}^{\prime}(s)\left(\partial_{x} \eta_{1}\right)^{2}+g_{2}^{\prime}(s)\left(\partial_{x} \eta_{2}\right)^{2}+g_{3}^{\prime}(s)\left(\partial_{x} \eta_{3}\right)^{2}\right) \mathrm{d} s \mathrm{~d} x \\
& +c_{4} \int_{0}^{L} \int_{0}^{+\infty}\left(g_{1}(s)\left(\partial_{x} \eta_{1}\right)^{2}+g_{2}(s)\left(\partial_{x} \eta_{2}\right)^{2}+g_{3}(s)\left(\partial_{x} \eta_{3}\right)^{2}\right) \mathrm{d} s \mathrm{~d} x .
\end{aligned}
$$

On the other hand, using (2.13) and definition of $E, I_{i}$ and $L$, there exists a positive constant $N_{4}$ (not depending on $N_{1}$ ) such that

$$
\left(N_{1}-N_{4}\right) E \leq L \leq\left(N_{1}+N_{4}\right) E,
$$

then, choosing $N_{1}>\max \left\{2 c_{4}, N_{4}\right\}$ and using the fact that $g_{i}^{\prime} \leq 0$, we get

$$
L^{\prime}(t) \leq-c_{3} E(t)+c_{4} \int_{0}^{L}\left[\int_{0}^{+\infty}\left(g_{1}(s)\left(\partial_{x} \eta_{1}\right)^{2}+g_{2}(s)\left(\partial_{x} \eta_{2}\right)^{2}+g_{3}(s)\left(\partial_{x} \eta_{3}\right)^{2}\right) \mathrm{d} s\right] \mathrm{d} x .
$$

In order to finish the proof of the stability, we need to estimate the last three terms in the right hand of (4.20). Inspired by [14], we have the following lemma.

Lemma 4.6 ([14]) For $i=1,2,3$, there exist positive constants $d_{i}$ and $\tilde{d}_{i}$ such that, for any $\epsilon_{0}>0$, then we have the following inequalities:

$$
\int_{0}^{L} \int_{0}^{+\infty} g_{i}(s)\left(\partial_{x} \eta_{i}\right)^{2} \mathrm{~d} s \mathrm{~d} x \leq-d_{i} E^{\prime}(t), \text { if (2.18) holds }
$$

and

$$
G^{\prime}\left(\epsilon_{0} E(t)\right) \int_{0}^{L}\left(\int_{0}^{+\infty} g_{i}(s)\left(\partial_{x} \eta_{i}\right)^{2} \mathrm{~d} s\right) \mathrm{d} x \leq-\tilde{d}_{i} E^{\prime}(t)+\tilde{d}_{i} \epsilon_{0} E(t) G^{\prime}\left(\epsilon_{0} E(t)\right),
$$

if (2.20) holds and (2.18) does not hold.

Proof. The proof of this lemma is similar to the proof of Lemma 3.3 in [14] and is omitted.

Now, going back to the proof of Theorem 2.2, if (2.18) holds for all $i \in\{1,2,3\}$, then (4.20) and (4.21) imply that

$$
L^{\prime}(t) \leq-c_{3} E(t)-c_{4}\left(d_{1}+d_{2}+d_{3}\right) E^{\prime}(t) .
$$

Let $F(t)=L(t)+c_{4}\left(d_{1}+d_{2}+d_{3}\right) E(t)$, combining (4.19) with (4.23), we have $F^{\prime}(t) \leq-c^{\prime} F(t)$, where

$$
c^{\prime}=\frac{c_{3}}{N_{1}+N_{4}+c_{4}\left(d_{1}+d_{2}+d_{3}\right)} .
$$

Integrating over $(0, t)$ and using (4.19), we obtain (2.22) with

$$
c^{\prime \prime}=\frac{F(0)}{N_{1}-N_{4}+c_{4}\left(d_{1}+d_{2}+d_{3}\right)} .
$$


Next, if (2.18) does not hold at least for one $i \in\{1,2,3\}$, then thanks to (4.21) and (4.22), we get that

$$
\begin{aligned}
G^{\prime}\left(\epsilon_{0} E(t)\right) \int_{0}^{L} \int_{0}^{+\infty} g_{i}(s)\left(\partial_{x} \eta_{i}\right)^{2} \mathrm{~d} s \mathrm{~d} x \leq & -\alpha_{i} G^{\prime}\left(\epsilon_{0} E(t)\right) E^{\prime}(t)-\beta_{i} E^{\prime}(t) \\
& +\epsilon_{0} \beta_{i} G^{\prime}\left(\epsilon_{0} E(t)\right) E^{\prime}(t)
\end{aligned}
$$

where

$$
\alpha_{i}= \begin{cases}d_{i}, & \text { if }(2.18) \text { holds } \\ 0, & \text { otherwise }\end{cases}
$$

and

$$
\beta_{i}= \begin{cases}0, & \text { if }(2.20) \text { holds } \\ \tilde{d}_{i}, & \text { otherwise }\end{cases}
$$

Multiplying (4.20) by $G^{\prime}\left(\epsilon_{0} E(t)\right)$, using $(4.24)$ and let $0<\epsilon_{0}<\frac{c_{3}}{c_{4}\left(\beta_{1}+\beta_{2}+\beta_{3}\right)}$, we obtain

$$
\begin{aligned}
& G^{\prime}\left(\epsilon_{0} E(t)\right) L(t)+c_{4}\left(\beta_{1}+\beta_{2}+\beta_{3}+\left(\alpha_{1}+\alpha_{2}+\alpha_{3}\right) G^{\prime}\left(\epsilon_{0} E(t)\right)\right) E^{\prime}(t) \\
\leq & -c_{5} E(t) G^{\prime}\left(\epsilon_{0} E(t)\right)
\end{aligned}
$$

where

$$
c_{5}=c_{3}-c_{4} \epsilon_{0}\left(\beta_{1}+\beta_{2}+\beta_{3}\right)
$$

Let

$$
F(t)=\kappa\left[G^{\prime}\left(\epsilon_{0} E(t)\right) L(t)+c_{4}\left(\beta_{1}+\beta_{2}+\beta_{3}+\left(\alpha_{1}+\alpha_{2}+\alpha_{3}\right) G^{\prime}\left(\epsilon_{0} E(t)\right)\right) E^{\prime}(t)\right],
$$

where $\kappa>0$. Using the fact that $G^{\prime}\left(\epsilon_{0} E(t)\right)$ is non-increasing and (4.25), we get

$$
F^{\prime}(t) \leq-c_{5} \tau E(t) G^{\prime}\left(\epsilon_{0} E(t)\right) .
$$

Combining (4.19) with the fact that $G^{\prime}\left(\epsilon_{0} E(t)\right) \leq G^{\prime}\left(\epsilon_{0} E(0)\right)$, we choose $\tau>0$ small enough such that

$$
F(t) \leq E(t) \quad \text { and } \quad F(0) \leq 1 .
$$

Then, choosing $c^{\prime}=c_{5} \tau$ (note that $s \mapsto s G^{\prime}\left(\epsilon_{0} s\right)$ is non-decreasing), we arrive at

$$
F^{\prime}(t) \leq-c^{\prime} F(t) G^{\prime}\left(\epsilon_{0} F(t)\right) .
$$

This implies that $(H(F(t)))^{\prime} \geq c^{\prime}$, where $H(t)$ is defined in (2.25). Then, integrating (4.27) over $(0, t)$, using $F(0) \leq 1, H(1)=0$ and $H(t)$ is decreasing, we get

$$
H(F(t)) \geq c^{\prime} t
$$

which implies $F(t) \leq H^{-1}\left(c^{\prime} t\right)$. Finally, the fact that $F(t) \sim E(t)$ gives $(2.24)$ with

$$
C^{\prime \prime}=\frac{1}{\tau c_{4}\left(\beta_{1}+\beta_{2}+\beta_{3}\right)},
$$

which completes the proof Theorem 2.2 . 


\section{References}

[1] F. Alabau Boussouira, J. E. Muñoz Rivera and D. S. Almeida Júnior, Stability to weak dissipative Bresse system, J. Math. Anal. Appl. 374 (2011), no. 2, 481-498.

[2] D. S. Almeida Júnior, M. L. Santos and J. E. Muñoz Rivera, Stability to 1-D thermoelastic Timoshenko beam acting on shear force, Z. Angew. Math. Phys. 65 (2014), no. 6, 1233-1249.

[3] F. Ammar-Khodja et al., Energy decay for Timoshenko systems of memory type, J. Differential Equations 194 (2003), no. 1, 82-115.

[4] T. A. Apalara, S. A. Messaoudi and A. A. Keddi, On the decay rates of Timoshenko system with second sound, Math. Methods Appl. Sci. 39 (2016), no. 10, 2671-2684.

[5] H. Brezis, Functional analysis, Sobolev spaces and partial differential equations, Universitext, Springer, New York (2011).

[6] B. D. Coleman, W. J. Hrusa and D. R. Owen, Stability of equilibrium for a nonlinear hyperbolic system describing heat propagation by second sound in solids, Arch. Rational Mech. Anal. 94 (1986), no. 3, 267-289.

[7] C. M. Dafermos, Asymptotic stability in viscoelasticity, Arch. Rational Mech. Anal. 37 (1970).

[8] R. Datko, J. Lagnese and M. P. Polis, An example on the effect of time delays in boundary feedback stabilization of wave equations, SIAM J. Control Optim. 24 (1986), no. 1, 152-156.

[9] L. H. Fatori and J. E. M. Rivera, Rates of decay to weak thermoelastic Bresse system, IMA J. Appl. Math. 75 (2010), no. 6, 881-904.

[10] A. Guesmia, Asymptotic stability of abstract dissipative systems with infinite memory, J. Math. Anal. Appl. 382 (2011), no. 2, 748-760.

[11] A. Guesmia, On the stabilization for Timoshenko system with past history and frictional damping controls, Palest. J. Math. 2 (2013), no. 2, 187-214.

[12] A. Guesmia and S. A. Messaoudi, On the control of a viscoelastic damped Timoshenko-type system, Appl. Math. Comput. 206 (2008), no. 2, 589-597.

[13] A. Guesmia and S. A. Messaoudi, General energy decay estimates of Timoshenko systems with frictional versus viscoelastic damping, Math. Methods Appl. Sci. 32 (2009), no. 16, $2102-2122$.

[14] A. Guesmia and M. Kafini, Bresse system with infinite memories, Math. Methods Appl. Sci. 38(2014),no. 11, 2389-2402.

[15] W. Liu, General decay of the solution for a viscoelastic wave equation with a time-varying delay term in the internal feedback, J. Math. Phys. 54 (2013), no. 4, 043504, 9 pp.

[16] J. E. Lagnese, G. Leugering and E. J. P. G. Schmidt, Modeling, analysis and control of dynamic elastic multi-link structures, Systems \& Control: Foundations \& Applications, Birkhäuser Boston, Inc., Boston, MA (1994). 
[17] V. Komornik, Exact controllability and stabilization, RAM: Research in Applied Mathematics, Masson, Paris, 1994.

[18] Z. Liu and B. Rao, Energy decay rate of the thermoelastic Bresse system, Z. Angew. Math. Phys. 60 (2009), no. 1, 54-69.

[19] M. Kirane, B. Said-Houari and M. N. Anwar, Stability result for the Timoshenko system with a time-varying delay term in the internal feedbacks, Commun. Pure Appl. Anal. 10 (2011), no. 2, 667-686.

[20] M. Kirane and B. Said-Houari, Existence and asymptotic stability of a viscoelastic wave equation with a delay, Z. Angew. Math. Phys. 62 (2011), no. 6, 1065-1082.

[21] J. E. Muñoz Rivera and R. Racke, Mildly dissipative nonlinear Timoshenko systems - global existence and exponential stability, J. Math. Anal. Appl. 276 (2002), no. 1, 248-278.

[22] S. Nicaise and C. Pignotti, Stability and instability results of the wave equation with a delay term in the boundary or internal feedbacks, SIAM J. Control Optim. 45 (2006), no. 5, $1561-1585$.

[23] S. Nicaise, C. Pignotti and J. Valein, Exponential stability of the wave equation with boundary time-varying delay, Discrete Contin. Dyn. Syst. Ser. S 4 (2011), no. 3, 693-722.

[24] A. Pazy, Semigroups of linear operators and applications to partial differential equations, Applied Mathematical Sciences, 44, Springer, New York, 1983.

[25] R. Racke, Thermoelasticity with second sound - exponential stability in linear and non-linear 1-d, Math. Methods Appl. Sci. 25 (2002), no. 5, 409-441.

[26] B. Said-Houari and Y. Laskri, A stability result of a Timoshenko system with a delay term in the internal feedback, Appl. Math. Comput. 217 (2010), no. 6, 2857-2869.

[27] B. Said-Houari, S. A. Messaoudi and A. Guesmia, General decay of solutions of a nonlinear system of viscoelastic wave equations, NoDEA Nonlinear Differential Equations Appl. 18 (2011), no. 6, 659-684.

[28] M. L. Santos, D. S. Almeida Júnior and J. E. Muñoz Rivera, The stability number of the Timoshenko system with second sound, J. Differential Equations 253 (2012), no. 9, 27152733.

[29] G. Q. Xu, S. P. Yung and L. K. Li, Stabilization of wave systems with input delay in the boundary control, ESAIM Control Optim. Calc. Var. 12 (2006), no. 4, 770-785. 